J. Anal. Math. (to appear)

\title{
ASYMPTOTIC BEHAVIOR OF POLYNOMIALS ORTHONORMAL ON A HOMOGENEOUS SET
}

\author{
Franz Peherstorfer And Peter Yuditskit
}

\begin{abstract}
Let $E$ be a homogeneous compact set, for instance a Cantor set of positive length. Further let $\sigma$ be a positive measure with $\operatorname{supp}(\sigma)=E$. Under the condition that the absolutely continuous part of $\sigma$ satisfies a Szegö-type condition we give an asymptotic representation, on and off the support, for the polynomials orthonormal with respect to $\sigma$. For the special case that $E$ consists of a finite number of intervals and that $\sigma$ has no singular component this is a nowaday well known result of Widom. If $E=[a, b]$ it becomes a classical result due to Szegö and in case that there appears in addition a singular component, it is due to KolmogorovKrein. In fact the results are presented for the more general case that the orthogonality measure may have a denumerable set of mass-points outside of $E$ which are supposed to accumulate on $E$ only and to satisfy (together with the zeros of the associated Stieltjes function) the free-interpolation Carleson-type condition. Up to the case of a finite number of mass points this is even new for the single interval case. Furthermore, as a byproduct of our representations, we obtain that the recurrence coefficients of the orthonormal polynomials behave asymptotically almost periodic. Or in other words the Jacobi matrices associated with the above discussed orthonormal polynomials are compact perturbations of a one-sided restriction of almost periodic Jacobi matrices with homogeneous spectrum. Our main tool is a theory of Hardy spaces of character-automorphic functions and forms on Riemann surfaces of Widom type, we use also some ideas of scattering theory for one-dimensional Schrödinger equations.
\end{abstract}

\section{INTRODUCTION}

Let $\sigma$ be a positive measure with a compact support. By $P_{n}(x)=P_{n}(x, \sigma)$ we denote the polynomial of degree $n$ orthonormal with respect to $\sigma$, i.e.:

$$
\int P_{n}(x) P_{m}(x) d \sigma(x)=\delta_{n, m} .
$$

It's well known that $\left\{P_{n}\right\}$ satisfies a three-term recurrence relation

$$
z P_{n}(z)=p_{n} P_{n-1}(z)+q_{n} P_{n}(z)+p_{n+1} P_{n+1}(z), \quad n=1,2, \ldots,
$$

This work was supported by the Austrian Science Fund FWF, project-number P12985-TEC

Typeset by $\mathcal{A} \mathcal{M} \mathcal{S}-\mathrm{T}_{\mathrm{E}} \mathrm{X}$ 
with initial data

$$
p_{0} P_{0}(z)=1, \quad z P_{0}(z)=q_{0} P_{0}(z)+p_{1} P_{1}(z) .
$$

One of the main problems is to find an explicit or at least an asymptotic representation of the orthonormal polynomials and their recurrence coefficients.

In the case when the support of the measure is the single interval, say $E=[-2,2]$, this problem has been solved by Szegö and Bernstein [30, 5] rather completely. As we shall see a crucial point was Szegö's discovery that the problem transformed to the unit circle by the well known conformal mapping $z: \mathbb{D}=\{\zeta:|\zeta|<1\} \rightarrow \overline{\mathbb{C}} \backslash E, z(\zeta)=\frac{1}{\zeta}+\zeta$ is closely connected with an extremal problem in the Hardy space $H^{2}$.

To be able to state Szegö's and our results we first will need some basic facts on Hardy spaces and functions of bounded characteristic [12]. if

Recall that a function $f(\zeta)$ meromorphic in $\mathbb{D}$ is said to be of bounded characteristic

$$
\sup _{0<r<1}\left\{\int_{\mathbb{T}} \log ^{+}|f(r t)| d m(t)\right\}<\infty
$$

where $\mathbb{T}=\{t:|t|=1\}$ and $d m$ is the Lebesgue measure on $\mathbb{T}$. It can be represented as a ratio of two holomorphic functions bounded in the disk, that is,

$$
f(\zeta)=\frac{f_{+}(\zeta)}{f_{-}(\zeta)}, \quad \sup _{\zeta \in \mathbb{D}}\left|f_{ \pm}(\zeta)\right| \leq 1
$$

Such functions have the representation

$$
f_{ \pm}(\zeta)=\prod \frac{\overline{\zeta_{l}^{ \pm}}}{\left|\zeta_{l}^{ \pm}\right|} \frac{\zeta_{l}^{ \pm}-\zeta}{1-\overline{\zeta_{l}^{ \pm}} \zeta} \exp \left\{i c^{ \pm}+\int_{\mathbb{T}} \frac{\zeta+t}{\zeta-t} d \tau^{ \pm}(t)\right\}
$$

where $\zeta_{l}^{ \pm} \in \mathbb{D}, c^{ \pm} \in \mathbb{R}$ and $d \tau^{ \pm}(t)$ are positive measures on $\mathbb{T}$. One can decompose $d \tau^{ \pm}(t)$ into the absolutely continuous $d \tau_{a . c .}^{ \pm}(t)$ and singular $d \tau_{s .}^{ \pm}(t)$ part. The factor

$$
f_{ \pm}^{\text {out }}(\zeta)=\exp \left\{i c^{ \pm}+\int_{\mathbb{T}} \frac{\zeta+t}{\zeta-t} d \tau_{a . c .}^{ \pm}(t)\right\}
$$

is called the outer part of the function $f_{ \pm}(\zeta)$. It is defined uniquely (up to unimodular constant) via the boundary values of the modulus of the given function, $d \tau_{a . c .}^{ \pm}(t)=$ $-\log \left|f_{ \pm}(t)\right| d m(t)$. The remaining part of the function is called the inner part. It contains the Blaschke product and the singular component. The function $f(\zeta)$ is of Smirnov class, (or Nevanlinna class $N^{+}$), if the denominator $f_{-}$is an outer function.

As usual, $H^{p}$ denotes the Hardy space of functions $f(\zeta)$ analytic on $\mathbb{D}$ with

$$
\|f\|_{p}=\sup _{0<r<1}\left\{\int_{\mathbb{T}}|f(r t)|^{p} d m(t)\right\}^{\frac{1}{p}}<\infty .
$$

Note that any function from $H^{p}$ is a function of Smirnov class, and that functions of Smirnov class obey the maximum principle in the following form: if $f$ is of Smirnov class and $f(t) \in L^{p}, t \in \mathbb{T}$, then $f \in H^{p}$. 
Now we are ready to state Szegö's result in a suitable way. Let the transformed measure $\sigma^{*}(t)$, given by

$$
2 \int_{E=[-2,2]} f(x) d \sigma(x)=\int_{\mathbb{T}} f(z(t)) d \sigma^{*}(t),
$$

have a decomposition of the form

$$
d \sigma^{*}(t)=\rho(t) d m(t)+d \sigma_{s .}^{*}(t),
$$

where $\sigma_{s .}^{*}$ is a singular measure and $\rho(t)$ satisfies the so-called Szegö condition

$$
\int_{\mathbb{T}} \log \rho(t) d m(t)>-\infty .
$$

Then $\rho$ has a representation of the form

$$
\rho(t)=|D(t)|^{2} \quad \text { a.e. on } \mathbb{T},
$$

where

$$
D(\zeta):=\exp \left\{\frac{1}{2} \int_{\mathbb{T}} \log \rho(t) \frac{t+\zeta}{t-\zeta} d m(t)\right\}
$$

is an outer function. Since it is a characteristic property of an outer function, that

$$
\operatorname{clos}_{L^{2}}\left\{D H^{\infty}\right\}=H^{2},
$$

we have

$$
\nu(\rho):=\inf \left\{\int_{\mathbb{T}}|f(t)|^{2} \rho(t) d m(t): f \in H^{\infty}, \quad f(0)=1\right\}=D^{2}(0)
$$

with an extremal function

$$
f(\zeta, \rho)=\frac{D(0)}{D(\zeta)}
$$

Using the fact that the polynomials orthonormal on the unit circle with respect to the weight function $\rho$ form a so-called minimizing sequence for problem (0.5) (see e.g. [22]) Szegö has shown that uniformly on compact subsets of $\Omega=\overline{\mathbb{C}} \backslash E$ the asymptotic relation

$$
P_{n}(z(\zeta), \sigma) \sim \frac{\zeta^{-n}}{D(\zeta)}
$$

holds. By (0.6) it follows that the recurrence coefficients have the asymptotic behavior

$$
p_{n} \rightarrow 1 \quad \text { and } \quad q_{n} \rightarrow 0 \quad(n \rightarrow \infty) .
$$

Szegö also has given an asymptotic representation of the orthonormal polynomials on $\mathbb{T}$ with respect to $L^{2}$-norm.

Up to the next major step in a general characterization, due to Akhiezer-Tomchuk [1, $3,32]$ and Widom [34], it took almost 40 years. For the case of finite number of intervals, say $E:=\left[b_{0}, a_{0}\right] \backslash \cup_{j=1}^{N}\left(a_{j}, b_{j}\right)$ Akhiezer and Tomchuk derived so called comparative 
asymptotics, that is, the weight functions are divided into classes and if the asymptotic behavior of the orthonormal polynomials with respect to one weight function is known the asymptotics with respect to all other weight functions from this class can be given. At the end of the sixties Widom discovered the important fact that in case of several arcs and curves, moreover of a finite number of intervals, the extremal problem (0.5) has to be replaced by a much more sophisticated one which we are going to discuss next.

To see better the parallels to Szegö's theory let us uniformize the domain $\Omega=\overline{\mathbb{C}} \backslash E$ by the so called universal covering map $z(\zeta)$. Recall that $z(\zeta)$ is a meromorphic function which maps $\mathbb{D}$ onto $\Omega$ and which is automorphic with respect to the associated Fuchsian group $\Gamma$, i.e., $z(\gamma(\zeta))=z(\zeta), \forall \gamma \in \Gamma$, and any two preimages of $z_{0} \in \Omega$ are $\Gamma$-equivalent, i.e., $z\left(\zeta_{1}\right)=z\left(\zeta_{2}\right) \Rightarrow \exists \gamma \in \Gamma: \zeta_{1}=\gamma\left(\zeta_{2}\right)$. We normalize $z(\zeta)$ by the conditions $z(0)=\infty$, $(\zeta z)(0)>0$.

Furthermore, a character of $\Gamma$ is a complex-valued function $\alpha: \Gamma \rightarrow \mathbb{T}$, satisfying

$$
\alpha\left(\gamma_{1} \gamma_{2}\right)=\alpha\left(\gamma_{1}\right) \alpha\left(\gamma_{2}\right) \quad\left(\gamma_{1}, \gamma_{2} \in \Gamma\right)
$$

The characters form an Abelian compact group denoted by $\Gamma^{*}$.

For a given character $\alpha \in \Gamma^{*}$ define the space of character-automorphic functions

$$
H^{\infty}(\Gamma, \alpha)=\left\{f \in H^{\infty}: f(\gamma(\zeta))=\alpha(\gamma) f(\zeta), \forall \gamma \in \Gamma\right\}
$$

The Blaschke product

$$
b(\zeta)=b(\zeta, 0)=\prod_{\gamma \in \Gamma} \frac{\gamma(0)-\zeta}{1-\overline{\gamma(0)} \zeta} \frac{|\gamma(0)|}{\gamma(0)}
$$

is called the Green's function of $\Gamma$ with respect to the origin. Since it is a characterautomorphic function, there exists a $\mu \in \Gamma^{*}$ such that

$$
b(\gamma(\zeta))=\mu(\gamma) b(\zeta)
$$

Note, if $G(z)=G(z, \infty)$ denotes the Green's function of the domain $\Omega$, then

$$
G(z(\zeta), z(0))=-\log |b(\zeta, 0)|
$$

Without loss of generality we assume in this paper that $(b z)(0)=1$, i.e., the capacity of $E$ is equal to 1 .

Next let us assume for a moment that $\sigma(x)$ is absolutely continuous on $E$, i.e., $d \sigma(x)=$ $\sigma_{a . c}^{\prime}(x) d x$. Transforming this measure by the universal $z(t)$, we get

$$
\begin{aligned}
2 \int_{E} f(x) \sigma_{a . c .}^{\prime}(x) d x & =\int_{\mathbb{E}} f(z(t)) \sigma_{a . c .}^{\prime}(z(t))\left|z^{\prime}(t)\right| 2 \pi d m(t) \\
& =\int_{\mathbb{E}} f(z(t)) \rho(t) d m(t),
\end{aligned}
$$


where $\mathbb{E}$ is a fundamental set for the action of $\Gamma$ on $\mathbb{T}$. Under the conditions that $E$ consists of a finite number of intervals and that $\rho(t)$ satisfies the generalized Szegö condition (0.3) Widom has shown that the minimum problem, $\rho \in L_{d m \mid \mathbb{E}}^{1}$,

$$
\nu(\rho, \alpha):=\inf \left\{\int_{\mathbb{E}}|f(t)|^{2} \rho(t) d m(t): f \in H^{\infty}(\Gamma, \alpha) \text { and } f(0)=1\right\}
$$

and its unique extremal function $f(\zeta, \rho, \alpha)$ (which does not belong to $H^{\infty}$ in general) play a crucial role in the asymptotics of polynomials $\left\{P_{n}\right\}$ orthonormal on a finite number of intervals $E$. Indeed, he proved that the minimum deviation has the following asymptotic behavior

$$
p_{0}^{2} \ldots p_{n}^{2}=\min _{a_{i}} \int_{E}\left|x^{n}+a_{1} x^{n-1}+\cdots+a_{n}\right|^{2} d \sigma(x) \sim \nu\left(\rho, \mu^{n}\right)
$$

and uniformly on compact subsets of $\Omega$ the orthonormal polynomial is given asymptotically by

$$
\left(p_{0} \ldots p_{n}\right) P_{n}(z(\zeta), \sigma) b^{n}(\zeta) \sim f\left(\zeta, \rho, \mu^{n}\right)
$$

He gave also asymptotics on the support. Widom used spaces of multivalued functions on $\Omega$, but we presented his results in a way suitable in what follows.

Thus after Akhiezer-Tomchuk's, Widom's and subsequent investigations [4, 13, 18, 24-27, 33] the following natural questions arise:

a) Does there still hold an asymptotic representation like (0.6) respectively (0.11) if $\overline{\mathbb{C}} \backslash E$ is infinite connected, in particular, if $E$ is a Cantor type set?

b) Is there an analog of the Krein-Kolmogorov-Szegö Theorem, that is, does the asymptotic representation still hold if the measure has an arbitrary singular part on $E$ ?

c) How does the asymptotic representation change if outside of $E$ a denumerable set of mass-points is added?

d) Finally, what could be said on the asymptotic behavior of the recurrence coefficient?

Let us point out that so far the answer to the questions b)-d) have been unknown partly even for the case of a finite number of intervals. More precisely the answer to question b) is known for the case of one interval only [22, Sect. 2]. Concerning question c) only the case of a finite number of mass points could be handled (see [21]).

What concerns question d) partial answers are known (see $[4,18,24,25])$ when the support of the measure consists of a finite number of intervals. Indeed, it's well known nowadays that the recurrence coefficients behave asymptotically periodic, if the harmonic measure of each component of $E$ is rational, or in other words if the set $E$ can be described as the inverse image of a polynomial map. If the harmonic measure of at least one of the intervals of $E$ is not rational then it is conjectured that the recurrence coefficients behave uniformly almost periodic in the limit. Recall, that a sequence of real numbers $\left\{p_{n}\right\} \in l^{\infty}(\mathbb{Z})$ is called uniformly almost periodic if the set of sequences $\left\{\left\{p_{n+n_{k}}\right\}, n_{k} \in \mathbb{Z}\right\}$ is precompact in $l^{\infty}(\mathbb{Z})$. The general way to produce a sequence of this type looks as follows: let $\mathcal{G}$ be a compact Abelian group, and let $f(g)$ be a continuous function on $\mathcal{G}$, then

$$
p_{n}:=f\left(g_{0}+n g_{1}\right), \quad g_{0}, g_{1} \in \mathcal{G},
$$


is an almost periodic sequence. If $E$ consists of two intervals for rational weights with square root singularities at the boundary points, so called Bernstein-Szegö type weights, the almost periodic recurrence coefficients can be given even explicitly in terms of elliptic functions $[2,24]$. In the case of several intervals for this class of weight functions it would be still possible to give a representation in terms of Theta-functions of several variables, using results and methods given in $[11,31]$.

If the weight function satisfies a Szegö-type condition only we could derive from Widom's result (we will not carry out this because it's included in our more general results below), that the recurrence coefficients have the following representation

$$
p_{n}=p_{n}^{(1)}+p_{n}^{(2)}, \quad q_{n}=q_{n}^{(1)}+q_{n}^{(2)}, \quad n \in \mathbb{Z}_{+},
$$

where $\left\{p_{n}^{(1)}\right\},\left\{q_{n}^{(1)}\right\}$ are half-line restrictions of almost periodic sequences, and

$$
p_{n}^{(2)} \rightarrow 0, \quad q_{n}^{(2)} \rightarrow 0, \quad n \rightarrow \infty
$$

If we denote by $J^{(1)}$ the Jacobi matrix associated with $\left\{p_{n}^{(1)}\right\}$ and $\left\{q_{n}^{(1)}\right\}$, i.e.,

$$
\left(J^{(1)} u\right)_{n}=p_{n}^{(1)} u_{n-1}+q_{n}^{(1)} u_{n}+p_{n+1}^{(1)} u_{n+1}, \quad u \in l^{2}(\mathbb{Z}),
$$

(0.13) says that $J_{+}=J_{+}^{(1)}+J_{+}^{(2)}$ is a compact perturbation of the compression on $l^{2}\left(\mathbb{Z}_{+}\right)$of an almost periodic Jacobi matrix $J^{(1)}$. As usual, a Jacobi matrix is called almost periodic if the coefficient sequences are almost periodic.

In the last years the spectral theory of almost periodic Jacobi matrices has been studied extensively $[7,9,23]$ in particular in connection with integrable systems [11, 31]. For other new interesting developments concerning asymptotics of orthogonal polynomials see [10, 17]. Recently a complete description of almost periodic Jacobi matrices with homogeneous absolutely continuous spectrum has been given in [29] (see Theorem [29] below).

Following Carleson, we say that a compact set $E$ is homogeneous if there is an $\eta>0$ such that

$$
|(x-\delta, x+\delta) \cap E| \geq \eta \delta \quad \text { for all } 0<\delta<\operatorname{diam} E \quad \text { and all } x \in E,
$$

i.e., homogeneous sets are uniformly thick with respect to Lebesgue measure. For instance, Cantor sets of positive length are homogeneous (see Example in Sect. 1).

The following space of character-automorphic forms (see e.g. [36]) will play an important role.

Definition. Let $E$ be a homogeneous set and let $z: \mathbb{D} / \Gamma \equiv \Omega$ be a uniformization of the domain $\Omega=\overline{\mathbb{C}} \backslash E$. The space $A_{1}^{2}(\Gamma, \alpha)$ is formed by functions $f$, which are analytic on $\mathbb{D}$ and satisfy the following three conditions

1) $f$ is of Smirnov class

$$
\begin{aligned}
& \text { 2) } \frac{f(\gamma(\zeta))}{\gamma_{21} \zeta+\gamma_{22}}=\alpha(\gamma) f(\zeta) \quad \forall \gamma=\left[\begin{array}{ll}
\gamma_{11} & \gamma_{12} \\
\gamma_{21} & \gamma_{22}
\end{array}\right] \in \Gamma \\
& \text { 3) } \int_{\mathbb{E}}|f|^{2} d m<\infty
\end{aligned}
$$


where $\mathbb{E}$ is a fundamental set for the action of $\Gamma$ on $\mathbb{T}$.

Let us mention that in our context the space $A_{1}^{2}(\Gamma, \alpha)$ arises naturally in the following way. Since $\rho(t)$ from (0.3) is supposed to satisfy Szegö's condition (0.7) it can be represented in the form

$$
\rho(t)=|D(t)|^{2},
$$

where $D(\zeta)$ is an outer function. Since the measure $\rho(t) d m(t)$ is invariant with respect to the substitution $t \rightarrow \gamma(t)$, we have

$$
\left|\frac{D(\gamma(t))}{\gamma_{21} t+\gamma_{22}}\right|^{2}=|D(t)|^{2}
$$

and hence the outer function $D(\zeta)$ itself satisfies an automorphic property of the form

$$
\frac{D(\gamma(t))}{\gamma_{21} t+\gamma_{22}}=\beta(\gamma) D(t), \quad \beta \in \Gamma^{*} .
$$

$A_{1}^{2}(\Gamma, \alpha)$ is a closed subspace of $L_{d m \mid \mathbb{E}}^{2}$ with the reproducing kernel $k^{\alpha}\left(\zeta, \zeta_{0}\right)$ (the point evaluation functional is bounded):

$$
\left\langle f(t), k^{\alpha}\left(t, \zeta_{0}\right)\right\rangle=f\left(\zeta_{0}\right), \quad \zeta_{0} \in \mathbb{D}, f \in A_{1}^{2}(\Gamma, \alpha) .
$$

Put

$$
k^{\alpha}(\zeta)=k^{\alpha}(\zeta, 0) \quad \text { and } \quad K^{\alpha}(\zeta)=\frac{k^{\alpha}(\zeta)}{\sqrt{k^{\alpha}(0)}} .
$$

Theorem [29]. Let $E$ be a homogeneous set. Let $z: \mathbb{D} / \Gamma \equiv \overline{\mathbb{C}} \backslash E$ with the normalization $(b z)(0)=1$. Then the systems of functions $\left\{b^{n} K^{\alpha \mu^{-n}}\right\}_{n \in \mathbb{Z}_{+}}$and $\left\{b^{n} K^{\alpha \mu^{-n}}\right\}_{n \in \mathbb{Z}}$ form an orthonormal basis in $A_{1}^{2}(\Gamma, \alpha)$ and in $L_{d m \mid \mathbb{E}}^{2}$, respectively, for any $\alpha \in \Gamma^{*}$. With respect to this basis, the operator multiplication by $z(t)$ is a three-diagonal almost periodic Jacobi matrix, moreover

$$
z b^{n} K^{\alpha \mu^{-n}}=\mathcal{P}\left(\alpha \mu^{-n}\right) b^{n-1} K^{\alpha \mu^{-n+1}}+\mathcal{Q}\left(\alpha \mu^{-n}\right) b^{n} K^{\alpha \mu^{-n}}+\mathcal{P}\left(\alpha \mu^{-n-1}\right) b^{n+1} K^{\alpha \mu^{-n-1}},
$$

where

$$
\mathcal{P}(\alpha)=\left(\frac{K^{\alpha}}{K^{\alpha \mu}}\right)(0), \quad b^{\prime}(0) \mathcal{Q}(\alpha)=(z b)^{\prime}(0)+\left(\log \frac{K^{\alpha}}{K^{\alpha \mu}}\right)^{\prime}(0) .
$$

Conversely, every almost periodic Jacobi matrix (0.14) such that $\sigma(J)=\sigma_{\text {a.c. }}(J)=E$ can be represented in the form

$$
p_{n}=\mathcal{P}\left(\alpha^{-1} \mu^{n+1}\right), \quad q_{n-1}=\mathcal{Q}\left(\alpha^{-1} \mu^{n+1}\right),
$$

with some $\alpha \in \Gamma^{*}$.

Let us point out, that the recurrence coefficients $\left\{p_{n}\right\}$ and $\left\{q_{n}\right\}$ from (0.16) are uniformly almost periodic, since $\mathcal{P}(\alpha)$ and $\mathcal{Q}(\alpha)$ are continuous functions on the compact Abelian group $\Gamma^{*}$ (see $\left.(0.12)\right)$.

In this paper for homogeneous sets $E$ questions a)-d) will be answered. One of the main outputs of this paper is the following corollary of our Main Theorem. For simplicity here in the introduction we restrict ourselves to the case when no point-measures appear outside of $E$. 
Corollary 0.1. Let $\sigma$ be a positive measure, whose support is a homogeneous set $E$. Assume that $\log \sigma_{\text {a.c. }}^{\prime}(z(t)) \in L^{1}$ and define an outer function $D(\zeta), D(0)>0$, by the relation

$$
|D(t)|^{2}=2 \pi \sigma_{\text {a.c. }}^{\prime}(z(t))\left|z^{\prime}(t)\right|, \quad t \in \mathbb{T} .
$$

Then the minimum deviation and the orthonormal polynomials $P_{n}(z, \sigma)=\frac{z^{n}}{p_{0} \ldots p_{n}}+\ldots$ have the following asymptotic behavior $(n \rightarrow \infty)$

$$
\begin{gathered}
p_{0} \ldots p_{n} \sim \frac{D(0)}{K^{\beta \mu^{n}}(0)}, \\
P_{n}(z(\zeta)) \sim \frac{b^{-n}(\zeta) K^{\beta \mu^{n}}(\zeta)}{D(\zeta)}
\end{gathered}
$$

uniformly on each compact subset of $\overline{\mathbb{C}} \backslash E$ and

$$
D(t) P_{n}(z(t))-\left\{\left(b^{-n} K^{\beta \mu^{n}}\right)(t)+\frac{D(t)}{D(\bar{t})}\left(b^{-n} K^{\beta \mu^{n}}\right)(\bar{t})\right\} \rightarrow 0 \quad \text { in } L_{d m \mid \mathbb{E}}^{2}
$$

where $\beta$ and $\mu$ are given in (0.15) and (0.7), respectively.

For a comparison of this result with those one of Szegö and Widom (see (0.6), (0.10), (0.11)) let us note, that for a homogeneous set $E$ we have the following analogue of (0.4):

$$
\operatorname{clos}_{L_{d m \mid \mathbb{E}}^{2}}\left\{D H^{\infty}(\Gamma, \alpha)\right\}=A_{1}^{2}(\Gamma, \beta \alpha) .
$$

Thus the extremal function $f(\zeta, \rho, \alpha)$ in $(0.9), \rho$ given by $(0.8)$, is of the form

$$
f(\zeta, \rho, \alpha)=\frac{K^{\alpha \beta}(\zeta)}{K^{\alpha \beta}(0)} \frac{D(0)}{D(\zeta)},
$$

and hence

$$
\nu(\rho, \alpha)=\left[\frac{D(0)}{K^{\alpha \beta}(0)}\right]^{2} .
$$

But let us point out that neither the ideas of proof nor the methods of proof are related to Widom's paper [34], only the results can be considered as an extension of those one of Widom.

For the very special case $E=[-2,2]$ and thus $z(\zeta)=\zeta+1 / \zeta$ the Fuchsian group $\Gamma$ is trivial, i.e., $\Gamma=\{\operatorname{id}\}, \Gamma^{*}=\{1\}, b(\zeta)=\zeta, K^{\alpha}(\zeta) \equiv 1$. Hence the basis $\left\{b^{n} K^{\alpha \mu^{-n}}\right\}$ becomes the standard Fourier basis $\left\{t^{n}\right\}$ in $L^{2}$ and Corollary 0.1 becomes Szegö's result (in particular (0.19) becomes (0.6)).

What concerns (0.20), it reminds strongly to the well known formulas from scattering theory [19, 21]. Roughly speaking, the proof of our result is based on asymptotic orthogonality of "incoming" and "outgoing" subspaces. More precisely we prove a characterautomorphic analog of the following well known proposition:

$$
P_{+}\left\{f(t) t^{-n}\right\} \rightarrow 0, \quad n \rightarrow \infty,
$$


where $f \in L^{\infty}$ and $P_{+}$is the Riesz projection from $L^{2}$ onto $H^{2}$ (see Lemma 5.3).

Finally we would like to mention that even if $\sigma$ is absolutely continuous on $E$ and $\sigma^{\prime}$ satisfies the Szegö-type condition (0.3), then a one-dimensional perturbation of the corresponding Jacobi matrix may lead to a measure with a denumerable set of mass-points outside $E$. Therefore it is more natural to consider measures which may have a denumerable set of mass-points outside $E$, where the mass-points are supposed to accumulate on $E$ only. In fact, this point of view gives us more freedom and even helps us to prove our Main Theorem. If such mass-points appear then we assume that the set of poles (i.e. the set of mass-points) and zeros of the associated Stieltjes function have to satisfy the free-interpolation Carleson-type condition. Under these conditions asymptotics with respect to this wide class of measures are given. With the help of the new asymptotic representation the limit almost periodic behavior of the recurrence coefficients is proved.

The paper is organized as follows: First the necessary ingredients from the theory of Hardy spaces of character-automorphic functions and forms are given. In the second Section properties and a special representation of the Stieltjes function are presented. Using this representation in Section 3 we introduce an analogue of a scattering function and a transformation which plays a central role in the proof. It maps polynomials in character-automorphic forms. Further, the main ideas of the proof of the Main Theorem are briefly outlined. In Sections 4 and 5 we show that for a wide class of measures $\sigma$ this map is a bounded map from $L_{d \sigma}^{2}$ to $A_{1}^{2}(\Gamma, \alpha)$. Approximating a given measure by a sequence of such measures we are able to prove the Main Theorem and its Corollaries in the last Section.

Acknowledgment. We would like to thank V.A. Marchenko for helpful and stimulating discussions and M.Sodin for explanations of some properties of homogeneous sets.

\section{Preliminaries: the Hardy spaces on a Riemann surface of Widom type}

For a compact set $E \subset \mathbb{R}$ let us consider the open unit disk $\mathbb{D}$ as universal covering surface for the domain $\Omega=\overline{\mathbb{C}} \backslash E$. Thus there exists a meromorphic function $z(\zeta)$ mapping $\mathbb{D}$ onto $\Omega$ and a discrete subgroup $\Gamma$ of the group $S U(1,1)$ consisting of elements of the form

$$
\gamma=\left[\begin{array}{ll}
\gamma_{11} & \gamma_{12} \\
\gamma_{21} & \gamma_{22}
\end{array}\right], \gamma_{11}=\overline{\gamma_{22}}, \gamma_{12}=\overline{\gamma_{21}}, \operatorname{det} \gamma=1,
$$

such that $z$ is automorphic with respect to $\Gamma$, i.e., $z(\gamma(\zeta))=z(\zeta), \forall \gamma \in \Gamma$, and any two preimages of $z_{0} \in \Omega$ are $\Gamma$-equivalent, i.e.,

$$
z\left(\zeta_{1}\right)=z\left(\zeta_{2}\right) \Rightarrow \exists \gamma \in \Gamma: \zeta_{1}=\gamma\left(\zeta_{2}\right) .
$$

As usual let us define

$$
H^{\infty}(\Gamma)=\left\{f \in H^{\infty}: f \circ \gamma=f, \forall \gamma \in \Gamma\right\} .
$$

Note, if the space $H^{\infty}(\Gamma)$ is not trivial, i.e.,

$$
\exists f \in H^{\infty}(\Gamma): f(\zeta) \not \equiv f\left(\zeta_{0}\right),
$$


then the trajectory $\left\{\gamma\left(\zeta_{0}\right)\right\}_{\gamma \in \Gamma}$ satisfies the Blaschke condition. The Blaschke product

$$
b\left(\zeta, \zeta_{0}\right)=b\left(\zeta, \zeta_{0} ; \Gamma\right)=\prod_{\gamma \in \Gamma} \frac{\gamma\left(\zeta_{0}\right)-\zeta}{1-\overline{\gamma\left(\zeta_{0}\right)} \zeta} \frac{\left|\gamma\left(\zeta_{0}\right)\right|}{\gamma\left(\zeta_{0}\right)}
$$

is called the Green's function of $\Gamma$ with respect to $\zeta_{0}$.

If $G\left(z, z_{0}\right)$ denotes, as before, the Green's function of the domain $\Omega$, then

$$
G\left(z(\zeta), z\left(\zeta_{0}\right)\right)=-\log \left|b\left(\zeta, \zeta_{0}\right)\right| .
$$

The Green function is a character-automorphic function, that is there exists a $\mu_{\zeta_{0}} \in \Gamma^{*}$ such that $b\left(\gamma(\zeta), \zeta_{0}\right)=\mu_{\zeta_{0}}(\gamma) b\left(\zeta, \zeta_{0}\right)$. To simplify the notation we put

$$
b(\zeta)=b(\zeta, 0) \quad \text { and } \quad \mu=\mu_{0} .
$$

We will consider spaces of character-automorphic functions. For $\alpha \in \Gamma^{*}$, define

$$
H^{\infty}(\Gamma, \alpha)=\left\{f \in H^{\infty}: f \circ \gamma=\alpha(\gamma) f, \forall \gamma \in \Gamma\right\} .
$$

The domain $\overline{\mathbb{C}} \backslash E$ (respectively the group $\Gamma$ ) is said to be of Widom type if for any $\alpha \in \Gamma^{*}$ the space $H^{\infty}(\Gamma, \alpha)$ is not trivial, i.e. $H^{\infty}(\Gamma, \alpha) \neq\{$ const $\}[35,28]$. Also, in this case, $\Gamma$ acts dissipative on $\mathbb{T}$ with respect to $d m$, that is there exists a measurable (fundamental) set $\mathbb{E}$, which does not contain any two $\Gamma$-equivalent points, and the union $\cup_{\gamma \in \Gamma} \gamma(\mathbb{E})$ is a set of full measure [28].

Let $f$ be an analytic function in $\mathbb{D}, \gamma \in \Gamma$ and $k \in \mathbb{N}$. Then we put

It is easily verified that

$$
f \mid[\gamma]_{k}=\frac{f(\gamma(\zeta))}{\left(\gamma_{21} \zeta+\gamma_{22}\right)^{k}}
$$

$$
f\left|\left[\gamma_{1} \gamma_{2}\right]_{k}=\left(f \mid\left[\gamma_{1}\right]_{k}\right)\right|\left[\gamma_{2}\right]_{k} .
$$

Notice that $f \mid[\gamma]_{2}=f \forall \gamma \in \Gamma$, means that the form $f(\zeta) d \zeta$ is invariant with respect to the substitutions $\zeta \rightarrow \gamma(\zeta)(f(\zeta) d \zeta$ is an Abelian integral on $\mathbb{D} / \Gamma)$. Analogically to $A_{1}^{2}(\Gamma, \alpha)$ (see Introduction) for a group of Widom type we define the space $A_{2}^{1}(\Gamma, \alpha)$.

Definition. Let $\Gamma$ be a group of Widom type and let $\mathbb{E} \subset \mathbb{T}$ be a fundamental set. The space $A_{2}^{1}(\Gamma, \alpha)$ is formed by functions $f$, which are analytic on $\mathbb{D}$ and satisfy the following three conditions

$$
\begin{aligned}
& \text { 1) } f \text { is of Smirnov class } \\
& \text { 2) } f \mid[\gamma]_{2}=\alpha(\gamma) f \quad \forall \gamma \in \Gamma \\
& \text { 3) } \int_{\mathbb{E}}|f| d m<\infty .
\end{aligned}
$$

If $\Gamma$ is a group of Widom type then $A_{1}^{2}(\Gamma, \alpha)$ is a Hilbert space with the reproducing kernel $k^{\alpha}\left(\zeta, \zeta_{0}\right)$, moreover

$$
0<\inf _{\alpha \in \Gamma^{*}} k^{\alpha}\left(\zeta_{0}, \zeta_{0}\right) \leq \sup _{\alpha \in \Gamma^{*}} k^{\alpha}\left(\zeta_{0}, \zeta_{0}\right)<\infty .
$$

Let us also mention that the identity

$$
\operatorname{clos}_{L_{d m \mid \mathbb{E}}^{2}}\left\{D(t) H^{\infty}(\Gamma)\right\}=A_{1}^{2}(\Gamma, \alpha),
$$

where $D(t)$ is an outer function from $A_{1}^{2}(\Gamma, \alpha)$, generally speaking does not hold for an arbitrary group of this type. It is valid for groups of Widom type with Direct Cauchy Theorem. 
Theorem [14]. Let $\Gamma$ be a group of Widom type. The following statements are equivalent:

1) The function $K^{\alpha}(0)$ is continuous on $\Gamma^{*}$.

2) For $\alpha \in \Gamma^{*}$, let $\Delta^{\alpha}(\zeta) \in H^{\infty}(\Gamma, \alpha),\left\|\Delta^{\alpha}\right\| \leq 1$, be an extremal function of the problem

$$
\Delta^{\alpha}(0)=\sup \left\{|f(0)|: f \in H^{\infty}(\Gamma, \alpha),\|f\| \leq 1\right\} .
$$

Then $\Delta^{\alpha}(0) \rightarrow 1\left(\alpha \rightarrow 1_{\Gamma^{*}}\right)$.

3) The Direct Cauchy Theorem holds:

$$
\int_{\mathbb{E}} \frac{f}{b}(t) \frac{d t}{2 \pi i}=\frac{f}{b^{\prime}}(0), \quad \forall f \in A_{2}^{1}(\Gamma, \mu) .
$$

4) Let $\overline{t A_{1}^{2}\left(\Gamma, \alpha^{-1}\right)}=\left\{g=\overline{t f}: f \in A_{1}^{2}\left(\Gamma, \alpha^{-1}\right)\right\}$. Then

$$
L_{d m \mid \mathbb{E}}^{2}=\overline{t A_{1}^{2}\left(\Gamma, \alpha^{-1}\right)} \oplus A_{1}^{2}(\Gamma, \alpha) \quad \forall \alpha \in \Gamma^{*} .
$$

5) Every invariant subspace $M \subset A_{1}^{2}(\Gamma, \alpha)$ (i.e. $f M \subset M \forall f \in H^{\infty}(\Gamma)$ ) is of the form

$$
M=s A_{1}^{2}\left(\Gamma, \sigma^{-1} \alpha\right)
$$

for some character-automorphic inner function $s \in H^{\infty}(\sigma)$.

Definition [6]. A measurable set $E$ is homogeneous if there is an $\eta>0$ such that

$$
|(x-\rho, x+\rho) \cap E| \geq \eta \rho \quad \text { for all } 0<\rho<\operatorname{diam} E \quad \text { and all } x \in E .
$$

Example. Let us demonstrate that Cantor sets of positive length are homogeneous. First recall the construction of such a set [20]. We start with an interval of the length $l_{0}$ and take a sequence of numbers

$$
\left\{\varkappa_{j}\right\}_{j \geq 1}: \quad 0<\varkappa_{j}<1, \sum_{j \geq 1} \varkappa_{j}<\infty .
$$

At the first step we remove an open segment, whose length is $\varkappa_{1}$ part of the common length of the initial interval, so that on either side there remains a closed line segment of the length $l_{1}=\frac{1}{2}\left(1-\varkappa_{1}\right) l_{0}$. Then we make the same procedure with each of the remaining intervals, taking out $\varkappa_{2}$ part of each of them. Continuing in this way, we get a Cantor set $E=E\left(l_{0} ; \varkappa_{1}, \varkappa_{2}, \ldots\right)$, with the common length

$$
\left|E\left(l_{0} ; \varkappa_{1}, \varkappa_{2}, \ldots\right)\right|=\prod_{j \geq 1}\left(1-\varkappa_{j}\right) l_{0}>0 .
$$

Let us mention that this set consists of two Cantor sets of the form $E\left(l_{1} ; \varkappa_{2} \ldots\right)$, with $l_{1}=\frac{1}{2}\left(1-\varkappa_{1}\right) l_{0}$, or of four sets of the form $E\left(l_{2} ; \varkappa_{3}, \ldots\right), l_{2}=\frac{1}{2}\left(1-\varkappa_{2}\right) l_{1}$, and so on, $\ldots$, of $2^{n}$ sets of the form $E\left(l_{n} ; \varkappa_{n+1}, \ldots\right), l_{n}=\frac{1}{2}\left(1-\varkappa_{n}\right) l_{n-1}$. 
Now we check that

$$
|(x-\rho, x+\rho) \cap E| \geq \frac{\prod_{j \geq 1}\left(1-\varkappa_{j}\right)}{2} \rho, \quad \forall x \in E, \forall \rho \leq \operatorname{diam} E .
$$

Let $\frac{1}{2} \operatorname{diam} E \leq \rho \leq \operatorname{diam} E$, and $x \in E$. Then the interval $(x-\rho, x+\rho)$ contains at least one of the set $E\left(l_{1} ; \varkappa_{2}, \ldots\right)$. So,

$$
\begin{aligned}
|(x-\rho, x+\rho) \cap E| & \geq\left|E\left(l_{1} ; \varkappa_{2}, \ldots\right)\right|=\prod_{j \geq 2}\left(1-\varkappa_{j}\right) \cdot\left(1-\varkappa_{1}\right) \frac{l_{0}}{2} \\
& =\frac{\prod_{j \geq 1}\left(1-\varkappa_{j}\right)}{2} l_{0} \geq \frac{\prod_{j \geq 1}\left(1-\varkappa_{j}\right)}{2} \rho .
\end{aligned}
$$

If $\frac{1}{4} \operatorname{diam} E \leq \rho \leq \frac{1}{2} \operatorname{diam} E$ and $x \in E$, then the interval $(x-\rho, x+\rho)$ contains at least one of the set $E\left(l_{2} ; \varkappa_{3}, \ldots\right)$. Finally, if $\frac{1}{2^{n}} \operatorname{diam} E \leq \rho \leq \frac{1}{2^{n-1}} \operatorname{diam} E$ and $x \in E$, then the interval $(x-\rho, x+\rho)$ contains at least one of the set $E\left(l_{n} ; \varkappa_{n+1}, \ldots\right)$. Hence,

$$
|(x-\rho, x+\rho) \cap E| \geq\left|E\left(l_{n} ; \varkappa_{n+1}, \ldots\right)\right|=\frac{\prod_{j \geq 1}\left(1-\varkappa_{j}\right)}{2^{n}} l_{0} \geq \frac{\prod_{j \geq 1}\left(1-\varkappa_{j}\right)}{2} \rho .
$$

The following sufficient condition of homogeneity was proposed and proved by M. Sodin based on [8].

Proposition (M. Sodin). Let $E=\left[b_{0}, a_{0}\right] \backslash \cup_{j \geq 1}\left(a_{j}, b_{j}\right)$. Let $l_{j}=b_{j}-a_{j}, j \geq 1$ and put, formally, $l_{0}=1$. Let $\rho_{j, k}$ be the distance between the two gaps $\left(a_{j}, b_{j}\right)$ and $\left(a_{k}, b_{k}\right)$, $j \neq k, j, k \geq 1$ and let $\rho_{j, 0}$ denote the distance from the gap $\left(a_{j}, b_{j}\right)$ to the boundary of the interval $\left[b_{0}, a_{0}\right]$. If

$$
\sup _{j} \sum_{k \neq j} \frac{l_{j}^{1 / 2} l_{k}^{1 / 2}}{\rho_{j, k}}<\infty
$$

then $E$ is a homogeneous set.

Let us mention that the case of Julia sets of polynomials which are real and thus (up to the classical Chebyshev polynomials and its conjugates) of Cantor type are not homogeneous because the length of the Julia set is zero.

Theorem. Let $E$ be a homogeneous set, then $\overline{\mathbb{C}} \backslash E$ is of Widom type and the Direct Cauchy Theorem holds.

The proof of this theorem is based mainly on the following lemma of Jones and Marshall [16], who following Carleson, considered the Corona problem for the surface $\overline{\mathbb{C}} \backslash E$, where $E$ is a homogeneous set.

Lemma. Let $E=\left[b_{0}, a_{0}\right] \backslash \cup_{j \geq 1}\left(a_{j}, b_{j}\right)$ be a homogeneous set. From each interval $\left(a_{j}, b_{j}\right)$ let us pick arbitrarily exactly one point $x_{j}$. Then there is a constant $N$ which depends only on the value $\eta$ in (1.1), such that

$$
\sup _{j \geq 1} \sum_{i \neq j} G\left(x_{i}, x_{j}\right) \leq N<\infty
$$

To finish this section, we would like to extend the DCT to functions with infinitely many poles. 
Lemma 1.1. Let $B \in H^{\infty}(\Gamma, \alpha)$ be a Blaschke product, $B=\prod_{l} b\left(\zeta, \zeta_{l}\right)$, and $f \in A_{2}^{1}(\Gamma, \alpha)$. If

$$
\sum_{l}\left|\frac{f\left(\zeta_{l}\right)}{B^{\prime}\left(\zeta_{l}\right)}\right|<\infty
$$

then

$$
\int_{\mathbb{E}} \frac{f}{B} \frac{d t}{2 \pi i}=\sum_{l} \frac{f\left(\zeta_{l}\right)}{B^{\prime}\left(\zeta_{l}\right)}
$$

Proof. In the proof we use a Poisson-like kernel. Let $K^{\beta}\left(\zeta, \zeta_{l}\right)=\frac{k^{\beta}\left(\zeta, \zeta_{l}\right)}{\sqrt{k^{\beta}\left(\zeta_{l}, \zeta_{l}\right)}}$. Then

$$
1=\int_{\mathbb{E}}\left|K^{\beta}\left(t, \zeta_{l}\right)\right|^{2} d m=\int_{\mathbb{E}} K^{\beta}\left(t, \zeta_{l}\right)\left(\overline{t K^{\beta}\left(t, \zeta_{l}\right)}\right) \frac{d t}{2 \pi i} .
$$

Let us show that

$$
\overline{t K^{\beta}\left(t, \zeta_{l}\right)}=\frac{K^{\beta^{-1}} \mu_{\zeta_{l}}\left(t, \zeta_{l}\right)}{b\left(t, \zeta_{l}\right)} \frac{b^{\prime}\left(\zeta_{l}, \zeta_{l}\right)}{\left|b^{\prime}\left(\zeta_{l}, \zeta_{l}\right)\right|}
$$

The function $\frac{K^{\beta^{-1} \mu_{\zeta_{l}}\left(\zeta, \zeta_{l}\right)}}{b\left(\zeta, \zeta_{l}\right)}$ is orthogonal to $A_{1}^{2}\left(\Gamma, \beta^{-1}\right)$, and, hence, it is of the form $\frac{K^{\beta^{-1} \mu_{\zeta_{l}}\left(t, \zeta_{l}\right)}}{b\left(t, \zeta_{l}\right)}=\overline{t g_{0}}, g_{0} \in A_{1}^{2}(\Gamma, \beta)$. But, due to DCT,

$$
\begin{aligned}
\left\langle g, g_{0}\right\rangle & =\left\langle\overline{t g_{0}}, \overline{t g}\right\rangle=\left\langle\frac{K^{\beta^{-1} \mu_{\zeta_{l}}\left(t, \zeta_{l}\right)}}{b\left(t, \zeta_{l}\right)}, \overline{t g}\right\rangle \\
& =\int_{\mathbb{E}} \frac{K^{\beta^{-1} \mu_{\zeta_{l}}\left(t, \zeta_{l}\right)}}{b\left(t, \zeta_{l}\right)} g(t) \frac{d t}{2 \pi i}=\frac{K^{\beta^{-1} \mu_{\zeta_{l}}\left(\zeta_{l}, \zeta_{l}\right)}}{b^{\prime}\left(\zeta_{l}, \zeta_{l}\right)} g\left(\zeta_{l}\right) .
\end{aligned}
$$

Therefore,

$$
g_{0}=k^{\beta}\left(t, \zeta_{l}\right) \overline{\left(\frac{K^{\beta^{-1} \mu_{\zeta_{l}}\left(\zeta_{l}, \zeta_{l}\right)}}{b^{\prime}\left(\zeta_{l}, \zeta_{l}\right)}\right)}=K^{\beta}\left(t, \zeta_{l}\right) \frac{K^{\beta}\left(\zeta_{l}, \zeta_{l}\right) K^{\beta^{-1}} \mu_{\zeta_{l}}\left(\zeta_{l}, \zeta_{l}\right)}{\left|b^{\prime}\left(\zeta_{l}, \zeta_{l}\right)\right|} \frac{b^{\prime}\left(\zeta_{l}, \zeta_{l}\right)}{\left|b^{\prime}\left(\zeta_{l}, \zeta_{l}\right)\right|}
$$

And, since $\left\|g_{0}\right\|=1$, we get (1.2), and the identity

$$
\frac{K^{\beta}\left(\zeta_{l}, \zeta_{l}\right) K^{\beta^{-1} \mu_{\zeta_{l}}\left(\zeta_{l}, \zeta_{l}\right)}}{\left|b^{\prime}\left(\zeta_{l}, \zeta_{l}\right)\right|}=1
$$

Put

$$
P^{\beta}\left(t, \zeta_{l}\right)=\frac{K^{\beta}\left(t, \zeta_{l}\right) K^{\beta^{-1}} \mu_{\zeta_{l}}\left(t, \zeta_{l}\right)}{b\left(t, \zeta_{l}\right)} \frac{b^{\prime}\left(\zeta_{l}, \zeta_{l}\right)}{\left|b^{\prime}\left(\zeta_{l}, \zeta_{l}\right)\right|} .
$$

As it was shown $P^{\beta}\left(t, \zeta_{l}\right) \frac{d t}{2 \pi i} \geq 0$, and $\int_{\mathbb{E}} P^{\beta}\left(t, \zeta_{l}\right) \frac{d t}{2 \pi i}=1$. 
For the given functions $B$ and $f$ consider the series

$$
\tilde{f}=\sum_{l} \frac{f\left(\zeta_{l}\right)}{B^{\prime}\left(\zeta_{l}\right)} P^{\beta}\left(t, \zeta_{l}\right) B
$$

with some $\beta \in \Gamma^{*}$. This series converges absolutely in $A_{2}^{1}(\Gamma, \alpha)$ and interpolates the function $f$ since

$$
\tilde{f}\left(\zeta_{l}\right)=\frac{f\left(\zeta_{l}\right)}{B^{\prime}\left(\zeta_{l}\right)} \frac{K^{\beta}\left(\zeta_{l}, \zeta_{l}\right) K^{\beta^{-1}} \mu_{\zeta_{l}}\left(\zeta_{l}, \zeta_{l}\right)}{b^{\prime}\left(\zeta_{l}, \zeta_{l}\right)} \frac{b^{\prime}\left(\zeta_{l}, \zeta_{l}\right)}{\left|b^{\prime}\left(\zeta_{l}, \zeta_{l}\right)\right|} B^{\prime}\left(\zeta_{l}\right)=f\left(\zeta_{l}\right)
$$

So, the function $f$ can be represented in the form: $f=\tilde{f}+B g$, with $g \in A_{2}^{1}(\Gamma)$. Due to DCT

$$
\int_{\mathbb{E}} \frac{f}{B} \frac{d t}{2 \pi i}-\int_{\mathbb{E}} \frac{\tilde{f}}{B} \frac{d t}{2 \pi i}=\int_{\mathbb{E}} \frac{f-\tilde{f}}{B} \frac{d t}{2 \pi i}=0
$$

and since the series converges absolutely we can integrate it term by term, which proves the lemma.

\section{A special Representation of the Stieltues function}

For the following we need some additional notation. Let $E$ be a homogeneous set and $X \subset \mathbb{R} \backslash E$ be a set of points which can accumulate only to the set $E$. Let $\sigma$ be a positive measure with support $E \cup X$. To this measure we associate the so-called Stieltjes function with a special normalization

$$
r(z)=1+\int \frac{d \sigma(x)}{x-z}=1+\sum_{x_{l} \in X} \frac{\sigma_{l}}{x_{l}-z}+\int_{E} \frac{d \sigma(x)}{x-z} .
$$

It is a function meromorphic on $\Omega=\overline{\mathbb{C}} \backslash E$ and such that

$$
\frac{r(z)-\overline{r(z)}}{z-\bar{z}} \geq 0
$$

The function $-1 / r(z)$ possesses the same properties, and therefore it has the representation

$$
-1 / r(z)=-1+\int \frac{d \sigma^{(\tau)}(x)}{x-z}=-1+\sum_{x_{l}^{(\tau)} \in X^{(\tau)}} \frac{\sigma_{l}^{(\tau)}}{x_{l}^{(\tau)}-z}+\int_{E} \frac{d \sigma^{(\tau)}(x)}{x-z} .
$$

The support of the measure $d \sigma^{(\tau)}(x)$ is the set $E \cup X^{(\tau)}$, where $X^{(\tau)}$ is the set of zeros of the function $r(z)$.

The following property of the measures $\sigma$ and $\sigma^{(\tau)}$ plays an essential role in what follows. 
Lemma 2.1. Suppose that the measures $\sigma$ and $\sigma^{(\tau)}$ are related by (2.1) and (2.2). Then the polynomial map

$$
P^{(\tau)}(z)=P(z)-\int \frac{P(x)-P(z)}{x-z} d \sigma(x)
$$

induces a unitary map from $L_{d \sigma}^{2}$ to $L_{d \sigma(\tau)}^{2}$. Furthermore, the inverse operator has the form

$$
P(z)=P^{(\tau)}(z)+\int \frac{P^{(\tau)}(x)-P^{(\tau)}(z)}{x-z} d \sigma^{(\tau)}(x) .
$$

Proof. Let $J$ be the Jacobi matrix associated with the measure $\sigma$,

$$
\int \frac{d \sigma(x)}{x-z}=\left\langle(J-z)^{-1} e, e\right\rangle,
$$

where

$$
J=\left[\begin{array}{cccc}
q_{0} & p_{1} & 0 & 0 \\
p_{1} & q_{1} & p_{2} & 0 \\
0 & p_{2} & q_{3} & \ddots \\
0 & 0 & \ddots & \ddots
\end{array}\right] \quad \text { and } e=\left[\begin{array}{c}
p_{0} \\
0 \\
0 \\
\vdots
\end{array}\right] .
$$

Direct calculation shows that

$$
\int \frac{d \sigma^{(\tau)}(x)}{x-z}=\left\langle\left(J^{(\tau)}-z\right)^{-1} e, e\right\rangle,
$$

where

$$
J^{(\tau)}=J+e\langle, e\rangle .
$$

Therefore, orthonormal polynomials with respect to the measure $\sigma^{(\tau)}$ satisfy the same recurrence relation $(0.2)$ but with initial data

$$
p_{0} P_{0}^{(\tau)}(z)=1, \quad z P_{0}^{(\tau)}=\left(q_{0}+p_{0}^{2}\right) P_{0}^{(\tau)}+P_{1}^{(\tau)} .
$$

For this reason (2.3) transforms the system of orthonormal polynomials corresponding to the measure $\sigma$ into the system of orthonormal polynomials with respect to the measure $\sigma^{(\tau)}$.

The same arguments show that (2.4) is the inverse map.

The following theorem describes quite general properties of a function $r(z(\zeta))$ as a function on the universal covering.

Theorem [29]. Assume that the set $X$ of poles of $r(z)$ satisfies the condition

$$
\sum_{x_{l} \in X} G\left(x_{l}\right)<\infty .
$$

then $r(z(\zeta))$ is a function of bounded characteristic without a singular component.

We will use essentially a special representation of $r(z(\zeta))$, which follows from this Theorem. 
Lemma 2.2. Let $r(z)$ be a function of the form (2.1). Suppose that $X$ satisfies condition (2.5) and assume that $\log \sigma_{\text {a.c. }}^{\prime}(z(t)) \in L^{1}$.

Then $r(z)$ has a representation of the form

$$
r(z(\zeta))=\frac{\psi(\zeta)}{\phi(\zeta)},
$$

where $\psi$ and $\phi$ are of Smirnov class; $\psi|[\gamma]=\alpha(\gamma) \psi, \phi|[\gamma]=\alpha(\gamma) \phi$ with some $\alpha \in \Gamma^{*}$ and

$$
\psi(t) \overline{\phi(t)}-\phi(t) \overline{\psi(t)}=-t z^{\prime}(t), \quad t \in \mathbb{T} .
$$

Proof. We would like to define an outer part of $\phi$ by the relation

$$
|\phi|^{2} \operatorname{Im} r \circ z=-\frac{t z^{\prime}}{2 i} .
$$

It is known that $z^{\prime}$ is of bounded characteristic, moreover $b^{2} z^{\prime}$ is the outer function. Since $\frac{1}{\pi} \operatorname{Im} r=\sigma_{a . c .}^{\prime}$ and $\log \sigma_{\text {a.c. }}^{\prime}(z(t)) \in L^{1}$, an outer function $\phi^{\text {out }}$ is well defined by (2.8).

Since $\operatorname{Im} r \circ z$ is an automorphic function, and since

$$
\left|\frac{z^{\prime} \circ \gamma}{\left(\gamma_{21} t+\gamma_{22}\right)^{2}}\right|=\left|z^{\prime} \circ \gamma\right|\left|\gamma^{\prime}\right|=\left|z^{\prime}\right|,
$$

we have

$$
\left|\frac{\phi^{\text {out }} \circ \gamma}{\gamma_{21} t+\gamma_{22}}\right|^{2}=\left|\phi^{\text {out }}\right|^{2} .
$$

Due to the uniqueness property of an outer function

$$
\frac{\phi^{\text {out }} \circ \gamma}{\gamma_{21} t+\gamma_{22}}=\alpha_{\text {out }}(\gamma) \phi^{\text {out }}
$$

with some $\alpha_{\text {out }} \in \Gamma^{*}$. Let $B_{X}(\zeta)$ denote the Blaschke product with zeros at $\left\{\zeta_{l}: z\left(\zeta_{l}\right) \in\right.$ $X\}$. Due to condition (2.5) it is well defined. Now we put $\phi(\zeta):=B_{X}(\zeta) \phi^{\text {out }}(\zeta)$. This is a function of bounded characteristic without a singular component, possessing the automorphic property we need. Next, let us put $\psi=(r \circ z) \phi$. This function has no poles, and, due to the cited Theorem, it also does not have a singular component. Thus the lemma is proved.

Remark. We would like to point out that the function $\phi$ depends only on the absolutely continuous part of the measure $\sigma_{\text {a.c. }}$ on $E$ and on the support of the measure $\sigma$ outside $E$, i.e. on the set $X$. We will see that all asymptotics are given only in terms of these functions.

By the way, the outer function $D$ in (0.17) and the function $\phi$ are related in the following way $|D|=\left|z^{\prime} / \phi\right|$. So, under the normalization $D(0)>0$ and $\phi^{\text {out }}(0)>0$, we have

$$
\frac{1}{D(\zeta)}=\left(\frac{\phi^{\text {out }}}{\left(-z^{\prime}\right) b^{2}}\right)(\zeta)
$$

Besides the additive representation (2.1) the function $r(z)$ possesses the following exponential (or multiplicative) representation. 
Lemma 2.3. Let $r(z)$ be a function of the form (2.1). Denote by $x_{k}^{(\tau)} \in X^{(\tau)}$ the nearest right-hand side zero to the pole $x_{k} \in X$. Then

$$
r(z)=\exp \left\{\frac{1}{\pi} \int \frac{f(x) d x}{x-z}\right\},
$$

where

$$
f(x)=\left\{\begin{array}{c}
\pi, x_{k}<x<x_{k}^{(\tau)} \\
u(x), x \in E \\
0, \text { otherwise }
\end{array}\right.
$$

and $u(x)=\arg r(x), x \in E$.

Moreover, if (2.5) holds, then $\log \sigma_{\text {a.c. }}^{\prime}(z(t)) \in L^{1}$ if and only if $\log \sin u(z(t)) \in L^{1}$.

Proof. The representation (2.10) follows immediately from the general exponential representation of functions with positive imaginary part in the upper half-plane:

$$
r(z)=\text { Const } \cdot \exp \left\{\frac{1}{\pi} \int_{\mathbb{R}} \arg r(x+i 0)\left(\frac{1}{x-z}-\frac{x}{1+x^{2}}\right) d x\right\}
$$

We only have to mention, that outside $E$ the function $r(z)$ is real, so the argument of $r(z)$ here is equal to 0 or $\pi$ and note that $r(\infty)=1$.

Splitting the integral into two parts we have

$$
\begin{aligned}
r(z) & =\exp \left\{\sum \int_{x_{k}}^{x_{k}^{(\tau)}} \frac{d x}{x-z}\right\} \cdot \exp \left\{\frac{1}{\pi} \int_{E} u(x) \frac{d x}{x-z}\right\} \\
& =r_{1}(z) \cdot r_{2}(z) .
\end{aligned}
$$

The first factor has real boundary values on $E$, moreover they are positive, therefore $\operatorname{Im} r(x+i 0)=r_{1}(x+i 0) \cdot \operatorname{Im} r_{2}(x+i 0)$. Due to the Theorem mentioned before $r_{1} \circ z$ is of bounded characteristic, so $\log \left|r_{1} \circ z\right|=\log r_{1} \circ z \in L^{1}$.

Next let us consider the second factor. The function $\log r_{2}(z)$ is holomorphic in $\overline{\mathbb{C}} \backslash E$, its imaginary part

$$
U(z)=\frac{1}{\pi} \int_{E} \frac{\operatorname{Im} z}{|x-z|^{2}} u(x) d x
$$

is a bounded harmonic function. Consequently, $U \circ z$ is a bounded harmonic function in $\mathbb{D}$. Therefore the boundary values of the conjugated function $\log \left|r_{2} \circ z\right|$, for sure, belong to $L^{1}$. Now, $\operatorname{Im} r_{2}=\exp \left\{\log \left|r_{2}\right|\right\} \sin \left\{\arg r_{2}\right\}=\exp \left\{\log \left|r_{2}\right|\right\} \sin \{\arg r\}$. So $\log \sigma_{a . c}^{\prime} \circ z=$ $\log \left\{\frac{1}{\pi} \operatorname{Im} r \circ z\right\}$ is in $L^{1}$ if and only if $\log \sin \{u \circ z\}=\log \sin \{\arg r \circ z\}$ is in $L^{1}$.

The last lemma of this section gives us a sufficient condition such that the measure $\sigma$ has no singular component on $E$.

Lemma 2.4. Assume that (2.5) holds and that

$$
\int_{E}|r(x)| d x<\infty
$$


Then $\sigma_{s .} \mid E=0$.

Proof. Series expansion of $r(z)$ at infinity gives by $(2.1)$

$$
r(z)=1-\frac{p_{0}^{2}}{z}+\ldots,
$$

where

$$
p_{0}^{2}=\sum_{l} \sigma_{l}+\int_{E} d \sigma(x) .
$$

It suffices to show that

$$
p_{0}^{2}=\sum_{l} \sigma_{l}+\frac{1}{\pi} \int_{E} \operatorname{Im} r(x+i 0) d x .
$$

Since $B_{X} r \circ z \cdot b^{2} z^{\prime}$ is a function of Smirnov class, and due to (2.11) it is integrable we conclude that $B_{X} r \circ z \cdot b^{2} z^{\prime} \in A_{2}^{1}(\Gamma, \beta)$ with some $\beta \in \Gamma^{*}$. The differential $r(z) d z$ has poles at the points $x_{l}$ and at infinity. Taking in mind (2.1) and (2.12) we get, that the sum of residues of the given differential is equal to

$$
\sum \operatorname{Res} r(z) d z=-\sum_{l} \sigma_{l}+p_{0}^{2} .
$$

Since this series converges absolutely, using Lemma 1.1, we obtain

$$
\frac{1}{2 \pi i} \int_{\partial \Omega} r(z) d z=-\sum_{l} \sigma_{l}+p_{0}^{2} .
$$

Due to the symmetry property $\overline{r(\bar{z})}=r(z)$, we have $\operatorname{Re} r(x+i 0)=\operatorname{Re} r(x-i 0)$ and $\operatorname{Im} r(x+i 0)=-\operatorname{Im} r(x-i 0)$, hence

$$
\frac{1}{2 \pi i} \int_{\partial \Omega} r(z) d z=\frac{1}{\pi} \int_{E} \operatorname{Im} r(x+i 0) d x .
$$

Thus, (2.13) is proved.

\section{From sPeCtral DATA to SCATtering DATA}

In this Section we will first derive some properties of the following map

$$
h(\zeta)=h(\zeta, P ; \sigma)=\phi(\zeta) \int \frac{P(x)}{z(\zeta)-x} d \sigma(x),
$$

which will play an important role in what follows. Then we will briefly outline the main ideas of the proof of the Main Theorem given in Section 6 . 
The map $h$ from (3.1) can be represented in the form

$$
\begin{aligned}
h(\zeta, P ; \sigma) & =\phi(\zeta)\left\{\int \frac{P(x)-P(z(\zeta))}{z(\zeta)-x} d \sigma(x)+P(z(\zeta)) \int \frac{d \sigma(x)}{z(\zeta)-x}\right\} \\
& =\phi(\zeta)\left\{P(z(\zeta))-\int \frac{P(x)-P(z(\zeta))}{x-z(\zeta)} d \sigma(x)-P(z(\zeta)) r(z(\zeta))\right\} \\
& =\phi(\zeta) P^{(\tau)}(z(\zeta))-\psi(\zeta) P(z(\zeta)),
\end{aligned}
$$

as well as by Lemma 2.1

$$
h(\zeta)=h(\zeta, P ; \sigma)=\psi(\zeta) \int \frac{P^{(\tau)}(x)}{z(\zeta)-x} d \sigma^{(\tau)}(x) .
$$

With the help of these representations we obtain the following properties of the function $h: h \mid[\gamma]=\alpha(\gamma) h$, since the functions $\phi$ and $\psi$ have this property; $\mathrm{h}$ is of Smirnov class. Indeed, if $n=\operatorname{deg} P$, then $b^{n} P \in H^{\infty}$. So $b^{n} h(\zeta, P ; \sigma)$ is of Smirnov class. It remains to be shown, that $h$ has no poles at $\{\gamma(0)\}$. In fact, it has a zero at the origin. This follows immediately from (3.1). Since we can not guarantee, that $h$ is square-integrable on $\mathbb{E}$, in general $h$ does not belong to $A_{1}^{2}(\Gamma, \alpha)$.

Using transformation (3.1) we will pass from the standard spectral parameter, i.e., spectral measure $\sigma$, to a system of objects which remind to scattering data, i.e., to a unimodular function $s$ and a discrete measure $\nu$ supported outside of the essential spectrum (for the connection between scattering theory and orthogonal polynomials see [21]). What we are going to do in fact, it's only to rewrite a standard norm $\int|P|^{2} d \sigma$ in terms of the function $h$. Doing this at the last step we assume that $\sigma$ has no singular spectrum on $E$.

First let us express $P$ and $P^{(\tau)}$ in terms of the function $h$. Since $\phi(\bar{t})=\overline{\phi(t)}$ and $\psi(\bar{t})=\overline{\psi(t)}$, we get from $(3.2)$ :

$$
\left[\begin{array}{l}
h(t) \\
h(\bar{t})
\end{array}\right]=\left[\begin{array}{ll}
-\psi & \phi \\
-\bar{\psi} & \bar{\phi}
\end{array}\right]\left[\begin{array}{c}
P \\
P^{(\tau)}
\end{array}\right] .
$$

Therefore, using (2.6) we get

$$
t z^{\prime}\left[\begin{array}{c}
P \\
P^{(\tau)}
\end{array}\right]=\left[\begin{array}{ll}
\bar{\phi} & -\phi \\
\bar{\psi} & -\psi
\end{array}\right]\left[\begin{array}{l}
h(t) \\
h(\bar{t})
\end{array}\right],
$$

which gives the desired representation

$$
\left[\begin{array}{c}
\overline{\left(\frac{t z^{\prime}}{\phi}\right)} P \\
\overline{\left(\frac{t z^{\prime}}{\psi}\right)} P^{(\tau)}
\end{array}\right]=-\left[\begin{array}{cc}
1 & -s \\
1 & -s^{(\tau)}
\end{array}\right]\left[\begin{array}{c}
h(t) \\
\bar{t} h(\bar{t})
\end{array}\right],
$$

where $s=\phi /(\overline{t \phi}), s^{(\tau)}=\psi /(\overline{t \psi})$. 
Since $\sigma_{\text {a.c. }}^{\prime}(x)=\frac{1}{\pi} \operatorname{Im} r(x+i 0)$ it follows by $(2.7)$,

$$
\begin{aligned}
\int_{E}|P(x)|^{2} d \sigma_{a . c .}(x) & =\frac{1}{2} \int_{\mathbb{E}}|P(z(t))|^{2} \frac{1}{\pi}\left(\frac{-t z^{\prime}}{2 i|\phi|^{2}}\right)\left(z^{\prime} d t\right) \\
& =\frac{1}{2} \int_{\mathbb{E}}|P(z(t))|^{2} \frac{\left(-t z^{\prime}\right)\left(t z^{\prime}\right)}{|\phi|^{2}} d m,
\end{aligned}
$$

where $P$ is a polynomial. Substituting (3.3) in (3.4) we get for the absolutely continuous part of the measure $\sigma(x)$ :

$$
\int_{E}|P(x)|^{2} d \sigma_{a . c .}(x)=\int_{\mathbb{E}}\left|\frac{h(t)-s(t) \bar{t} h(\bar{t})}{\sqrt{2}}\right|^{2} d m(t) .
$$

Analogically, for the absolutely continuous part of the measure $d \sigma^{(\tau)}$, using (2.6), we get

$$
\int_{E}\left|P^{(\tau)}(x)\right|^{2} d \sigma_{a . c .}^{(\tau)}(x)=\int_{\mathbb{E}}\left|\frac{h(t)-s^{(\tau)}(t) \bar{t} h(\bar{t})}{\sqrt{2}}\right|^{2} d m(t) .
$$

For the pure point spectrum of $\sigma$ and $\sigma^{(\tau)}$ we also can pass from a polynomial $P(z)$ to the function $h(\zeta)=h(\zeta, P ; \sigma)$. Since $r=\psi / \phi$, we have $\sigma_{k}=-\left(\frac{\psi}{\phi^{\prime}} z^{\prime}\right)\left(x_{k}\right)$ and $\sigma_{k}^{(\tau)}=\left(\frac{\phi}{\psi^{\prime}} z^{\prime}\right)\left(x_{k}^{(\tau)}\right)$. In view of $(3.2)$,

$$
P\left(x_{k}\right)=-\frac{h}{\psi}\left(x_{k}\right) \quad \text { and } \quad P^{(\tau)}\left(x_{k}^{(\tau)}\right)=\frac{h}{\phi}\left(x_{k}^{(\tau)}\right)
$$

so

$$
\sum_{x_{k} \in X}\left|P\left(x_{k}\right)\right|^{2} \sigma_{k}=\sum_{x_{k} \in X}\left|\frac{h}{\psi}\right|^{2}\left(x_{k}\right) \sigma_{k}
$$

and

$$
\sum_{x_{k}^{(\tau)} \in X^{(\tau)}}\left|P^{(\tau)}\left(x_{k}^{(\tau)}\right)\right|^{2} \sigma_{k}^{(\tau)}=\sum_{x_{k}^{(\tau)} \in X^{(\tau)}}\left|\frac{h}{\phi}\right|^{2}\left(x_{k}^{(\tau)}\right) \sigma_{k}^{(\tau)} .
$$

Thus we can define discrete measures $\nu$ and $\nu^{(\tau)}$ on $\mathbb{D}$

$$
\nu\left(\zeta_{l}\right)=\frac{\sigma_{l}}{\left|\psi\left(\zeta_{l}\right)\right|^{2}}, \quad z\left(\zeta_{l}\right)=x_{l}
$$

and

$$
\nu^{(\tau)}\left(\zeta_{l}^{(\tau)}\right)=\frac{\sigma_{l}^{(\tau)}}{\left|\phi\left(\zeta_{l}^{(\tau)}\right)\right|^{2}}, \quad z\left(\zeta_{l}^{(\tau)}\right)=x_{l}^{(\tau)}
$$

These measures possess the automorphic property

$$
\left|\gamma_{21} \zeta_{l}+\gamma_{22}\right|^{2} \nu\left(\gamma\left(\zeta_{l}\right)\right)=\nu\left(\zeta_{l}\right), \quad\left|\gamma_{21} \zeta_{l}^{(\tau)}+\gamma_{22}\right|^{2} \nu^{(\tau)}\left(\gamma\left(\zeta_{l}^{(\tau)}\right)\right)=\nu\left(\zeta_{l}^{(\tau)}\right)
$$


Let $Z$ be a fundamental set in the support of the measure $\nu$. Then the sum

$$
\sum_{\zeta_{l} \in Z}\left|g\left(\zeta_{l}\right)\right|^{2} \nu\left(\zeta_{l}\right), \quad g \in A_{1}^{2}(\Gamma, \beta),
$$

in fact, does not depend on the choice of the fundamental set and

$$
\sum_{x_{k} \in X}\left|P\left(x_{k}\right)\right|^{2} \sigma_{k}=\sum_{\zeta_{l} \in Z}\left|h\left(\zeta_{l}\right)\right|^{2} \nu\left(\zeta_{l}\right) .
$$

Analogically,

$$
\sum_{x_{k}^{(\tau)} \in X^{(\tau)}}\left|P^{(\tau)}\left(x_{k}^{(\tau)}\right)\right|^{2} \sigma_{k}^{(\tau)}=\sum_{\zeta_{l}^{(\tau)} \in Z^{(\tau)}}\left|h\left(\zeta_{l}^{(\tau)}\right)\right|^{2} \nu^{(\tau)}\left(\zeta_{l}^{(\tau)}\right) .
$$

Hence, under the assumption $\sigma_{s} \mid E=0$, we get

$$
\int|P|^{2} d \sigma=\int_{\mathbb{E}}\left|\frac{h(t)-s(t) \bar{t} h(\bar{t})}{\sqrt{2}}\right|^{2} d m(t)+\sum_{\zeta_{l} \in Z}\left|h\left(\zeta_{l}\right)\right|^{2} \nu\left(\zeta_{l}\right)=:\|h\|_{s, \nu}^{2} .
$$

Now let us briefly and roughly outline the proof of the Main Theorem. Assume that $P(z) \rightarrow h(\zeta, P ; \sigma)$ is a bounded map from $L_{d \sigma}^{2}$ to $A_{1}^{2}(\Gamma, \alpha)$, i.e.,

$$
\int_{\mathbb{E}}|h|^{2} d m \leq C_{1} \int|P|^{2} d \sigma
$$

and that the measure $\nu$ possesses the property

$$
\sum_{\zeta_{l} \in Z}\left|h\left(\zeta_{l}\right)\right|^{2} \nu\left(\zeta_{l}\right) \leq C_{2} \int_{\mathbb{E}}|h|^{2} d m,
$$

then $\|\cdot\|_{s, \nu}^{2}$ in (3.10) gives us a norm in $A_{1}^{2}(\Gamma, \alpha)$ which is equivalent to the original one,

$$
\frac{1}{C_{1}}\|h\|^{2} \leq\|h\|_{s, \nu}^{2} \leq\left(2+C_{2}\right)\|h\|^{2} .
$$

Next let us consider the special system of functions $\left\{h_{n}(\zeta)\right\}$,

$$
h_{n}(\zeta)=h\left(\zeta, P_{n} ; \sigma\right) \text {, }
$$

which is important in proving our results, as we shall see in a moment. Note, that $h_{n} / \phi$ is the so called $n$-th function of second kind. The functions satisfy a three-term recurrence relation of the form $(0.2)$,

$$
z(\zeta) h_{n}(\zeta)=p_{n} h_{n-1}(\zeta)+q_{n} h_{n}(\zeta)+p_{n+1} h_{n+1}(\zeta),
$$


and by orthogonality of $P_{n}(0.1)$ and definition (3.1) it follows that $h_{n}(\zeta)$ has a zero of order $n+1$ at the origin. From this remark, and (3.13) we derive that

$$
\left(z^{n+1} h_{n}\right)(0)=p_{0} \ldots p_{n} \phi(0) .
$$

Thus, taking into consideration (3.10), we obtain that $\left\{h_{n}\right\}$ forms an orthonormal basis with respect to $\|\cdot\|_{s, \nu}$. So, to construct this system one can orthogonalize the system of functions

$$
h_{n}(\zeta)=\sum_{l \geq n} c_{l, n} b^{l+1}(\zeta) K^{\alpha \mu^{-(l+1)}}(\zeta) .
$$

In other words, $h_{n}$ is an extremal function of the problem:

$$
\sup \left\{|\tilde{h}(0)|^{2}: h=b^{(n+1)} \tilde{h}, \tilde{h} \in A_{1}^{2}\left(\Gamma, \alpha \mu^{-(n+1)}\right),\|h\|_{s, \nu}^{2} \leq 1\right\} .
$$

Let us compare the extremal problem (3.15) and the extremal problem

$$
\sup \left\{|\tilde{h}(0)|^{2}: h=b^{(n+1)} \tilde{h}, \tilde{h} \in A_{1}^{2}\left(\Gamma, \alpha \mu^{-(n+1)}\right),\|h\|^{2} \leq 1\right\},
$$

whose extremal function is evidently of the form $h=b^{n+1} K^{\alpha \mu^{-(n+1)}}$. We shall demonstrate in the next sections that

$$
\sum_{\zeta_{l} \in Z}\left|b^{n+1}\left(\zeta_{l}\right) K^{\alpha \mu^{-(n+1)}}\left(\zeta_{l}\right)\right|^{2} \nu\left(\zeta_{l}\right) \rightarrow 0, n \rightarrow \infty
$$

and

$$
P_{-}\left(\alpha^{-1}\right)\left\{\bar{s} b^{n+1} K^{\alpha \mu^{-(n+1)}}\right\} \rightarrow 0, \quad n \rightarrow \infty,
$$

where $P_{-}\left(\alpha^{-1}\right)$ is the orthogonal projection onto $L_{d m \mid \mathbb{E}}^{2} \ominus A_{1}^{2}\left(\Gamma, \alpha^{-1}\right)$. Now (3.17) says that $\left\langle\bar{s} b^{n+1} K^{\alpha \mu^{-(n+1)}}, \overline{t b^{n+1} K^{\alpha \mu^{-(n+1)}}}\right\rangle \rightarrow 0, n \rightarrow \infty$. Thus, using also (3.16), we get

$$
\left\|b^{n+1} K^{\alpha \mu^{-(n+1)}}\right\|_{s, \nu} \sim\left\|b^{n+1} K^{\alpha \mu^{-(n+1)}}\right\| .
$$

Similarly, $\left\langle b^{n+1} K^{\alpha \mu^{-(n+1)}}, h_{n}\right\rangle_{s, \nu} \sim\left\langle b^{n+1} K^{\alpha \mu^{-(n+1)}}, h_{n}\right\rangle$. It implies by (3.14)

$$
p_{0} \ldots p_{n} \phi(0) \sim K^{\alpha \mu^{-(n+1)}}(0),
$$

and

$$
h_{n} \sim b^{n+1} K^{\alpha \mu^{-(n+1)}},
$$

which gives by (3.3) an asymptotic relation for $P_{n}$. Recall that the asymptotic relations have been derived under the assumptions (3.11), (3.12). In the next two sections we will present a wide class of measures for which these assumptions are satisfied. Approximating a given measure by a sequence of such measures we are able to prove the Main Theorem. 


\section{Pure-Point spectrum in the Gaps}

In this section we investigate the pure-point spectrum of $\sigma$ and $\sigma^{(\tau)}$ on $\mathbb{R} \backslash E$.

Our goal is to present a sufficient condition such that the associated point measures $\nu$ and $\nu^{(\tau)}$ satisfy $(3.12)$.

Lemma 4.1. Let $r(z)$ be a function of the form (2.1), such that $\log \sigma_{\text {a.c. }}^{\prime}$ o $z \in L^{1}$. Assume that the set of zeros and poles $X \cup X^{(\tau)}$ of $r(z)$ satisfies

$$
\sup _{y_{l} \in X \cup X^{(\tau)}} \sum_{j \neq l} G\left(y_{j}, y_{l}\right) \leq-\log \delta \quad(0<\delta<1) .
$$

Then there exists a constant $C(E, \delta)<\infty$, such that $\forall \beta \in \Gamma^{*}$

$$
\sum_{x_{k} \in X}\left|\frac{g}{\psi}\right|^{2}\left(x_{k}\right) \sigma_{k} \leq C(E, \delta)\|g\|^{2}
$$

and

$$
\sum_{x_{k}^{(\tau)} \in X^{(\tau)}}\left|\frac{g}{\phi}\right|^{2}\left(x_{k}^{(\tau)}\right) \sigma_{k}^{(\tau)} \leq C(E, \delta)\|g\|^{2},
$$

where $g \in A_{1}^{2}(\Gamma, \beta), \overline{g(\bar{\zeta})}=g(\zeta), g(0)=0$.

Proof. We will prove (4.2), (4.3) could be proved in the same way. The proof is based on the following Theorem (see $[6,15]$ ): if $E$ is a homogeneous set, $\overline{\mathbb{C}} \backslash E \equiv \mathbb{D} / \Gamma$, then for any $\beta \in \Gamma^{*}$ there exists a $w \in H^{\infty}(\Gamma, \beta),\|w\| \leq 1$, such that $|w(\zeta)| \geq C(E)$. We may assume, that $\overline{w(\bar{\zeta})}=w(\zeta)$

Let $\left\{X^{N}\right\}$ be an exhaustion of $X$ by finite sets. Let $B_{N}$ be the Blaschke product with zeros in $\left\{\zeta_{j}: z\left(\zeta_{j}\right) \in X \cup X^{(\tau)} \backslash X^{N}\right\}, B_{N}(0)>0$. In this case $\overline{B_{N}(\bar{\zeta})}=B_{N}(\zeta)$. We note, that at any point $\zeta_{l}: z\left(\zeta_{l}\right) \in X^{N}$, we have $\left|B_{N}\left(\zeta_{l}\right)\right| \geq \delta$.

Let $w$ be a function from the above cited Theorem, such that the character of the function $B_{N} w g$ equals $\alpha$. The function $\frac{\left(B_{N} w g\right)^{2}}{\phi \psi} z^{\prime}$ has only a finite number of poles (all zeros of the product $\psi \phi$, except a finite number of them, were included in $B_{N}$ ). Thus, we are able to apply the (DCT):

$$
\int_{\mathbb{E}} \frac{\left(B_{N} w g\right)^{2}}{\phi \psi} z^{\prime} \frac{d t}{2 \pi i}=\sum_{z\left(\zeta_{l}\right) \in X^{N}}\left(\frac{B_{N} w g}{\psi}\right)^{2} \frac{\psi}{\phi^{\prime}} z^{\prime}
$$

The function $\left(\frac{B_{N} w g}{\psi}\right)$ is automorphic and real-valued on the real axis, therefore it defines a function on $\overline{\mathbb{C}} \backslash E$ which is real-valued on $\mathbb{R} \backslash E$. So, we can write square-module instead of square. Then, we get

$$
\sum_{z\left(\zeta_{l}\right) \in X^{N}}\left(\frac{B_{N} w g}{\psi}\right)^{2} \sigma_{l} \geq \delta^{2} C(E)^{2} \sum_{z\left(\zeta_{l}\right) \in X^{N}}\left|\frac{g}{\psi}\right|^{2} \sigma_{l} .
$$


On the other hand, taking into account, that $\left|\frac{z^{\prime}}{\phi \psi}\right|=\left|\frac{\psi \bar{\phi}-\phi \bar{\psi}}{\phi \psi}\right| \leq 2$, we get

$$
\left|\int_{\mathbb{E}} \frac{\left(B_{N} w g\right)^{2}}{\phi \psi} z^{\prime} \frac{d t}{2 \pi i}\right| \leq 2 \int_{\mathbb{E}}|g|^{2} d m
$$

Hence the lemma is proved.

Lemma 4.2. Let $\nu$ be a measure of the form (3.9) with the property

$$
\left\{\sum_{\zeta_{l} \in Z}\left|g\left(\zeta_{l}\right)\right|^{2} \nu\left(\zeta_{l}\right)\right\}^{1 / 2} \leq C\|g\|, \quad g \in A_{1}^{2}(\Gamma, \beta) .
$$

Then

$$
\left\{\sum_{\zeta_{l} \in Z}\left|\left(b^{n} K^{\alpha \mu^{-n}}\right)\left(\zeta_{l}\right)\right|^{2} \nu\left(\zeta_{l}\right)\right\}^{1 / 2} \rightarrow 0, \quad n \rightarrow \infty
$$

Proof. For fixed $\epsilon>0$ let

$$
\Gamma^{*}=\bigcup_{j=1}^{l(\epsilon)}\left\{\beta: \operatorname{dist}\left(\beta, \beta_{j}\right) \leq \eta(\epsilon)\right\}
$$

be a finite covering of $\Gamma^{*}$, such that

$$
2\left|1-\frac{\Delta^{\beta_{j}^{-1} \beta}(0) K^{\beta_{j}}(0)}{K^{\beta}(0)}\right| \leq \epsilon^{2}, \quad \operatorname{dist}\left(\beta, \beta_{j}\right) \leq \eta(\epsilon) .
$$

It means, that

$$
\left\|\left(\Delta^{\beta_{j}^{-1} \beta} K^{\beta_{j}}\right)-K^{\beta}\right\|^{2} \leq 1+1-2 \frac{\Delta^{\beta_{j}^{-1} \beta}(0) K^{\beta_{j}}(0)}{K^{\beta}(0)} \leq \epsilon^{2}, \quad \operatorname{dist}\left(\beta, \beta_{j}\right) \leq \eta(\epsilon) .
$$

For a fixed $\beta_{j}$ the sum

$$
\sum_{\zeta_{l} \in Z}\left|K^{\beta_{j}}\left(\zeta_{l}\right)\right|^{2} \nu\left(\zeta_{l}\right)<\infty
$$

is a convergent majorant for the series

$$
\sum_{\zeta_{l} \in Z}\left|\left(b^{n} K^{\beta_{j}}\right)\left(\zeta_{l}\right)\right|^{2} \nu\left(\zeta_{l}\right)
$$

Therefore, it tends to 0 as $n$ tends to infinity. It means, that there exists an $n_{0}$ such that

$$
\left\{\sum_{\zeta_{l} \in Z}\left|\left(b^{n} K^{\beta_{j}}\right)\left(\zeta_{l}\right)\right|^{2} \nu\left(\zeta_{l}\right)\right\}^{1 / 2} \leq \epsilon, \forall n>n_{0}, 1 \leq j \leq l(\epsilon) .
$$


Let, now, $n>n_{0}$ and $\beta_{j}: \operatorname{dist}\left(\beta_{j}, \alpha \mu^{-n}\right) \leq \eta(\epsilon)$. Then, due to (4.4) and (4.5) we get

$$
\begin{aligned}
& \left\{\sum_{\zeta_{l} \in Z}\left|\left(b^{n} K^{\alpha \mu^{-n}}\right)\left(\zeta_{l}\right)\right|^{2} \nu\left(\zeta_{l}\right)\right\}^{1 / 2} \\
\leq & \left\{\sum_{\zeta_{l} \in Z}\left|\left(b^{n} K^{\alpha \mu^{-n}}\right)\left(\zeta_{l}\right)-\left(b^{n} K^{\beta_{j}} \Delta^{\beta_{j}^{-1} \alpha \mu^{-n}}\right)\left(\zeta_{l}\right)\right|^{2} \nu\left(\zeta_{l}\right)\right\}^{1 / 2} \\
+ & \left\{\sum_{\zeta_{l} \in Z}\left|\left(b^{n} K^{\beta_{j}} \Delta^{\beta_{j}^{-1} \alpha \mu^{-n}}\right)\left(\zeta_{l}\right)\right|^{2} \nu\left(\zeta_{l}\right)\right\}^{1 / 2} \\
\leq & C\left\|K^{\alpha \mu^{-n}}-K^{\beta_{j}} \Delta^{\beta_{j}^{-1} \alpha \mu^{-n}}\right\|+\left\{\sum_{\zeta_{l} \in Z}\left|\left(b^{n} K^{\beta_{j}}\right)\left(\zeta_{l}\right)\right|^{2} \nu\left(\zeta_{l}\right)\right\}^{1 / 2} \\
\leq & (C+1) \epsilon .
\end{aligned}
$$

Combining these two lemmas we have the following proposition.

Lemma 4.3. Let $r(z)$ be a function of the form (2.1), such that $\log \sigma_{a . c .}^{\prime}{ }^{\circ} z \in L^{1}$. Assume that the system of zeros and poles $X \cup X^{(\tau)}$ of $r(z)$ satisfies (4.1). Then

$$
\sum_{\zeta_{l} \in Z}\left|b^{n}\left(\zeta_{l}\right) K^{\alpha \mu^{-n}}\left(\zeta_{l}\right)\right|^{2} \nu\left(\zeta_{l}\right) \rightarrow 0, n \rightarrow \infty
$$

and

$$
\sum_{\zeta_{l}^{(\tau)} \in Z^{(\tau)}}\left|b^{n}\left(\zeta_{l}^{(\tau)}\right) K^{\alpha \mu^{-n}}\left(\zeta_{l}^{(\tau)}\right)\right|^{2} \nu^{(\tau)}\left(\zeta_{l}^{(\tau)}\right) \rightarrow 0, n \rightarrow \infty,
$$

where $\nu$ and $\nu^{(\tau)}$ are defined by (3.7) and (3.8).

\section{Enclosure in $A_{1}^{2}(\Gamma, \alpha)$. Absolutely continuous spectrum}

In this section we present sufficient conditions for the map $P \rightarrow h(\zeta, P ; \sigma)$ to be a bounded map from $L_{d \sigma}^{2}$ to $A_{1}^{2}(\Gamma, \alpha)$.

With a given set of interlacing points $X \cup X^{(\tau)}$ from $\mathbb{R} \backslash E, E=\left[b_{0}, a_{0}\right] \backslash \cup_{j \geq 1}\left(a_{j}, b_{j}\right)$, we associate a special function $r_{0}(z)=r_{0}\left(z ; X \cup X^{(\tau)}\right)$ of the form (2.8) with the given set of poles and zeros by putting $u(x)=\pi / 2$. Let $d \sigma_{0}(x)=d \sigma_{0}\left(x ; X \cup X^{(\tau)}\right)$ be the measure associated with this function,

$$
1+\int \frac{d \sigma_{0}\left(x ; X \cup X^{(\tau)}\right)}{x-z}=r_{0}\left(z ; X \cup X^{(\tau)}\right)=\prod_{l} \frac{z-x_{l}^{(\tau)}}{z-x_{l}} \sqrt{\prod_{j \geq 0} \frac{z-a_{j}}{z-b_{j}}} .
$$


Lemma 5.1. Let $X \cup X^{(\tau)} \subset \mathbb{R} \backslash E$ be a given set of interlacing points, such that $X$ satisfies condition (2.5). Let the measure $d \sigma_{0}(z)=d \sigma_{0}\left(z ; X \cup X^{(\tau)}\right)$ be defined by (5.1).

Then $P \rightarrow h\left(\zeta, P ; \sigma_{0}\right)$ is a bounded map. Moreover

$$
\int_{\mathbb{E}}|h|^{2} d m \leq 2 \int P^{2} d \sigma_{0}
$$

Proof. First note, that $d \sigma_{0}$ is absolutely continuous on $E$. In fact, $r_{0}(x+i 0)$ takes pure imaginary values on $E$, so $\left|r_{0}(x)\right| d x=\operatorname{Im} r_{0}(x) d x$ and we can apply Lemma 2.4. Using Lemma 2.2, we present $r_{0}\left(z ; X \cup X^{(\tau)}\right)$ in the form $r_{0}=\psi_{0} / \phi_{0}$ with the property $(2.7)$. We put $s_{0}=\phi_{0} /\left(\overline{t \phi_{0}}\right), s_{0}^{(\tau)}=\psi_{0} /\left(\overline{t \psi_{0}}\right)$.

As a consequence of (3.5), (3.6), we obtain the estimate (see also Lemma 2.1):

$$
\begin{aligned}
& \left\langle\left[\begin{array}{cc}
1 & -s_{0} \\
1 & -s_{0}^{(\tau)}
\end{array}\right]\left[\begin{array}{c}
h(t) \\
\bar{t} h(\bar{t})
\end{array}\right],\left[\begin{array}{cc}
1 & -s_{0} \\
1 & -s_{0}^{(\tau)}
\end{array}\right]\left[\begin{array}{c}
h(t) \\
\bar{t} h(\bar{t})
\end{array}\right]\right\rangle_{L_{d m \mid \mathbb{E}}^{2}} \\
= & \int_{\mathbb{E}} P^{2}\left|\frac{t z^{\prime}}{\phi_{0}}\right|^{2} d m+\int_{\mathbb{E}}\left(P^{(\tau)}\right)^{2}\left|\frac{t z^{\prime}}{\psi_{0}}\right|^{2} d m \\
\leq & 2 \int_{E} P^{2} d \sigma_{0}+2 \int_{E}\left(P^{(\tau)}\right)^{2} d \sigma_{0}^{(\tau)} \\
= & 4 \int P^{2} d \sigma_{0} .
\end{aligned}
$$

Since

$$
\left[\begin{array}{cc}
1 & -s_{0} \\
1 & -s_{0}^{(\tau)}
\end{array}\right]^{*}\left[\begin{array}{cc}
1 & -s_{0} \\
1 & -s_{0}^{(\tau)}
\end{array}\right]=2\left[\begin{array}{cc}
\frac{1}{\frac{s_{0}+s_{0}^{(\tau)}}{2}} & -\frac{s_{0}+s_{0}^{(\tau)}}{2} \\
1
\end{array}\right]
$$

and $\left|\frac{s_{0}+s_{0}^{(\tau)}}{2}\right|^{2}+\left|\frac{s_{0}-s_{0}^{(\tau)}}{2}\right|^{2}=1$, we have

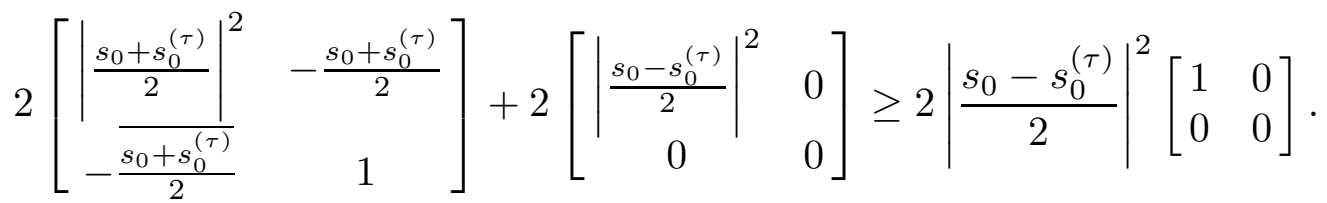

At last,

$$
\left|\frac{s_{0}-s_{0}^{(\tau)}}{2}\right|^{2}=\left|\frac{1-r_{0} / \bar{r}_{0}}{2}\right|^{2}=\sin ^{2}\left(\arg r_{0}\right)=1 .
$$

Combining (5.2), (5.3) and (5.4), we get

$$
2 \int_{\mathbb{E}}|h|^{2} d m \leq 4 \int P^{2} d \sigma_{0},
$$

which proves the assertion. 
Remark. Let us note, that in the same way it can be proved that the map $P \rightarrow h(\zeta, P, \sigma)$ is bounded if the associated Stieltjes function $r(z)$ possesses the property

$$
\delta \leq \arg r(x+i 0) \leq \pi-\delta, \quad x \in E
$$

with some $\delta>0$. In this case

$$
\int_{\mathbb{E}}|h|^{2} d m \leq \frac{2}{\sin ^{2} \delta} \int P^{2} d \sigma .
$$

Lemma 5.2. Let $X \cup X^{(\tau)}$ be a given set of interlacing points, such that $X$ satisfies condition (2.5). Let the measure $d \sigma_{0}(z)=d \sigma_{0}\left(z ; X \cup X^{(\tau)}\right)$ be defined by (5.1). Assume that the measure $d \sigma$ is equivalent to $d \sigma_{0}$, i.e.

$$
C_{1} d \sigma_{0} \geq d \sigma \geq C_{2} d \sigma_{0} .
$$

Then $P \rightarrow h(\zeta, P ; \sigma)$ is a bounded map. Moreover

$$
\int_{\mathbb{E}}|h|^{2} d m \leq 2 \frac{C_{1}}{C_{2}} \int P^{2} d \sigma .
$$

Proof. Let us represent $d \sigma$ in the form

$$
d \sigma=\frac{1}{w^{2}(x)} d \sigma_{0},
$$

where $1 / C_{1} \leq w^{2}(x) \leq 1 / C_{2}$. Define $W(\zeta)$ as an outer function with the given modulus of boundary values, $|W(t)|^{2}=w^{2}(z(t)), t \in \mathbb{T}$. In this case the functions $\phi$ and $\phi_{0}$ associated with $\sigma$ and $\sigma_{0}$, respectively, are related in the following way $\phi=W \phi_{0}$, and the function $h(\zeta, P ; \sigma)$ can be represented in the form

$$
h(\zeta, P ; \sigma)=h\left(\zeta, \frac{P}{w^{2}} ; \sigma_{0}\right) W(\zeta) .
$$

Therefore,

$$
\|h(\zeta, P ; \sigma)\|^{2} \leq \frac{1}{C_{2}}\left\|h\left(\zeta, \frac{P}{w^{2}} ; \sigma_{0}\right)\right\|^{2} \leq \frac{2}{C_{2}}\left\|\frac{P}{w^{2}}\right\|_{L_{d \sigma_{0}}^{2}}^{2} \leq 2 \frac{C_{1}}{C_{2}}\|P\|_{L_{d \sigma}^{2}}^{2} .
$$

Let us prove (3.17) not only for an individual function $f=s$, but for a family of functions $f_{\xi}$ depending continuously on a parameter $\xi$. 
Lemma 5.3. Let $\xi \mapsto f_{\xi}(t)$ be a $L^{\infty}\left(\alpha^{2}\right)$-valued continuous function on a compact set $\Xi$, $\left\|f_{\xi}\right\|_{L^{\infty}\left(\alpha^{2}\right)} \leq 1, \xi \in \Xi$. Then

$$
P_{-}\left(\alpha^{-1}\right)\left\{\bar{f}_{\xi} b^{n} K^{\alpha \mu^{-n}}\right\} \rightarrow 0, \quad n \rightarrow \infty, \text { uniformly on } \xi \in \Xi,
$$

where $P_{-}\left(\alpha^{-1}\right)$ is the orthogonal projection onto $L_{d m \mid \mathbb{E}}^{2} \ominus A_{1}^{2}\left(\Gamma, \alpha^{-1}\right)$.

Proof. For fixed $\epsilon>0$ let

$$
\Gamma^{*}=\bigcup_{j=1}^{l(\epsilon)}\left\{\beta: \operatorname{dist}\left(\beta, \beta_{j}\right) \leq \eta(\epsilon)\right\}
$$

be the same finite covering of $\Gamma^{*}$ as in the proof of Lemma 4.2, i.e.:

$$
\left\|\left(\Delta^{\beta_{j}^{-1} \beta} K^{\beta_{j}}\right)-K^{\beta}\right\| \leq \epsilon, \quad \operatorname{dist}\left(\beta, \beta_{j}\right) \leq \eta(\epsilon) .
$$

Let

$$
\Xi=\bigcup_{j^{\prime}=1}^{l^{\prime}(\epsilon)}\left\{\xi: \operatorname{dist}\left(\xi, \xi_{j^{\prime}}\right) \leq \eta^{\prime}(\epsilon)\right\}
$$

be a finite covering of $\Xi$, such that

$$
\left\|f_{\xi}-f_{\xi_{j^{\prime}}}\right\| \leq \epsilon, \quad \operatorname{dist}\left(\xi, \xi_{j^{\prime}}\right) \leq \eta^{\prime}(\epsilon) .
$$

For fixed $\beta$ and $\xi$ one can find $n_{0}$ such that

$$
\left\|P_{-}^{n}\left(\alpha^{-2} \beta\right) \bar{f}_{\xi} K^{\beta}\right\| \leq \epsilon, \forall n>n_{0}
$$

where $P_{-}^{n}(\alpha)$ is the orthogonal projection onto $L_{d m \mid \mathbb{E}}^{2} \ominus b^{-n} A_{1}^{2}\left(\Gamma, \alpha \mu^{n}\right)$. Therefore, there exists $n_{0}$ such that

$$
\left\|P_{-}^{n}\left(\alpha^{-2} \beta_{j}\right) \overline{f_{\xi_{j^{\prime}}}} K^{\beta_{j}}\right\| \leq \epsilon, \forall n>n_{0}, 1 \leq j \leq l(\epsilon), 1 \leq j^{\prime} \leq l^{\prime}(\epsilon) .
$$

From now on let $n>n_{0}=n_{0}(\epsilon)$. Let $\beta_{j}: \operatorname{dist}\left(\beta_{j}, \alpha \mu^{-n}\right) \leq \eta(\epsilon)$ and $\xi_{j^{\prime}}: \operatorname{dist}\left(\xi_{j^{\prime}}, \xi\right) \leq$ $\eta^{\prime}(\epsilon)$. For $h \in L_{d m \mid \mathbb{E}}^{2} \ominus A_{1}^{2}\left(\Gamma, \alpha^{-1}\right)$, we write

$$
\begin{aligned}
& \left\langle b^{n} K^{\alpha \mu^{-n}}, f_{\xi} h\right\rangle=\left\langle b^{n} K^{\alpha \mu^{-n}},\left(f_{\xi}-f_{\xi_{j^{\prime}}}\right) h\right\rangle \\
+ & \left\langle b^{n}\left(K^{\alpha \mu^{-n}}-\Delta^{\alpha \mu^{-n} \beta_{j}^{-1}} K^{\beta_{j}}\right), f_{\xi_{j^{\prime}}} h\right\rangle+\left\langle b^{n} \Delta^{\alpha \mu^{-n} \beta_{j}^{-1}} K^{\beta_{j}}, f_{\xi_{j^{\prime}}} h\right\rangle .
\end{aligned}
$$

Then

$$
\left|\left\langle b^{n} K^{\alpha \mu^{-n}},\left(f_{\xi}-f_{\xi_{j^{\prime}}}\right) h\right\rangle\right| \leq\left\|\left(f_{\xi}-f_{\xi_{j^{\prime}}}\right)\right\|\|h\|\left\|K^{\alpha \mu^{-n}}\right\| \leq \epsilon\|h\|
$$

and

$$
\left|\left\langle b^{n}\left(K^{\alpha \mu^{-n}}-\Delta^{\alpha \mu^{-n} \beta_{j}^{-1}} K^{\beta_{j}}\right), f_{\xi_{j^{\prime}}} h\right\rangle\right| \leq\left\|f_{\xi_{j^{\prime}}}\right\|\|h\|\left\|K^{\alpha \mu^{-n}}-\Delta^{\alpha \mu^{-n} \beta_{j}^{-1}} K^{\beta_{j}}\right\| \leq \epsilon\|h\| .
$$

And for the last term we have

$$
\begin{aligned}
& \left|\left\langle b^{n} \Delta^{\alpha \mu^{-n} \beta_{j}^{-1}} K^{\beta_{j}}, f_{\xi_{j^{\prime}}} h\right\rangle\right|=\left|\overline{f_{\xi_{j^{\prime}}}} K^{\beta_{j}}, b^{-n} \overline{\Delta^{\alpha \mu^{-n} \beta_{j}^{-1}}} h\right\rangle \mid \\
= & \left|\left\langle P_{-}^{n}\left(\alpha^{-2} \beta_{j}\right) \overline{f_{\xi_{j^{\prime}}}} K^{\beta_{j}}, b^{-n} \overline{\Delta^{\alpha \mu^{-n}} \beta_{j}^{-1}} h\right\rangle\right| \leq\|h\|\left\|P_{-}^{n}\left(\alpha^{-2} \beta_{j}\right) \overline{f_{\xi_{j^{\prime}}}} K^{\beta_{j}}\right\| \leq \epsilon\|h\| .
\end{aligned}
$$

Therefore, $\left|\left\langle P_{-}\left(\alpha^{-1}\right)\left\{\bar{f}_{\xi} b^{n} K^{\alpha \mu^{-n}}\right\}, h\right\rangle\right| \leq 3 \epsilon\|h\|$. Putting $h=P_{-}\left(\alpha^{-1}\right)\left\{\bar{f}_{\xi} b^{n} K^{\alpha \mu^{-n}}\right\}$, we get $\left\|P_{-}\left(\alpha^{-1}\right)\left\{\bar{f}_{\xi} b^{n} K^{\alpha \mu^{-n}}\right\}\right\| \leq 3 \epsilon$. 


\section{Main Theorem}

Theorem. Let $E$ be a homogeneous set and $X \subset \mathbb{R} \backslash E$ be a set of points which can accumulate only to the set $E$. Let $\sigma$ be a positive measure with the support $E \cup X$. Assume that $\log \sigma_{\text {a.c. }}^{\prime}(z(t)) \in L^{1}$ and that the set of poles $X$ and zeros $X^{(\tau)}$ of the Stieltjes function

$$
r(z)=1+\int \frac{d \sigma}{x-z}
$$

satisfies the condition

$$
\sup _{y_{l} \in X \cup X^{(\tau)}} \sum_{j \neq l} G\left(y_{j}, y_{l}\right)<\infty .
$$

Then the minimum deviation and the orthonormal polynomials $P_{n}(z, \sigma)=\frac{z^{n}}{p_{0} \ldots p_{n}}+\ldots$ have the following asymptotic behavior $(n \rightarrow \infty)$

$$
\begin{gathered}
\frac{K^{\alpha \mu^{-(n+1)}}(0)}{p_{0} \ldots p_{n} \phi(0)} \rightarrow 1, \\
\overline{\left(\frac{-t z^{\prime}}{\phi}\right)} P_{n}-\left\{b^{n+1} K^{\alpha \mu^{-(n+1)}}-s \overline{\left(t b^{n+1} K^{\alpha \mu^{-(n+1)}}\right)}\right\} \rightarrow 0 \quad \text { in } L_{d m \mid \mathbb{E}}^{2},
\end{gathered}
$$

and

$$
\left|P_{n} b^{n}-\frac{\phi}{\left(-z^{\prime}\right) b^{2}} K^{\alpha^{-1} \mu^{n+2}}\right| \rightarrow 0
$$

uniformly on each compact subset of $\overline{\mathbb{C}} \backslash E$. Here $\phi$ is chosen and $\alpha$ is given as in Lemma 2.2 and $s=\phi \overline{(t \phi)}$.

The proof of the theorem will be divided into several steps. The main part deals with the statement (6.2). First we show an upper estimate.

Lemma 6.1. Under the assumptions of the previous Theorem, we have

$$
\varlimsup_{n \rightarrow \infty} \frac{K^{\alpha \mu^{-(n+1)}}(0)}{p_{0} \ldots p_{n} \phi(0)} \leq 1 .
$$

Furthermore, (6.2) implies (6.3).

Proof. Consider the norm of the function in (6.3),

$$
\begin{aligned}
& \left\|\overline{\left(\frac{-t z^{\prime}}{\phi}\right)} P_{n}-\left\{b^{n+1} K^{\alpha \mu^{-(n+1)}}-s \overline{\left(t b^{n+1} K^{\alpha \mu^{-(n+1)}}\right)}\right\}\right\|^{2} \\
= & \int_{\mathbb{E}} P_{n}^{2}\left|\frac{t z^{\prime}}{\phi}\right|^{2} d m-2 \operatorname{Re}\left\langle b^{n+1} K^{\alpha \mu^{-(n+1)}}-s \overline{\left(t b^{n+1} K^{\alpha \mu^{-(n+1)}}\right)} \overline{\left(\frac{t z^{\prime}}{\phi}\right)} P_{n}\right\rangle \\
+ & \left\|b^{n+1} K^{\alpha \mu^{-(n+1)}}-s \overline{\left(t b^{n+1} K^{\alpha \mu^{-(n+1)}}\right)}\right\|^{2} .
\end{aligned}
$$


To prove (6.5) we only use the fact that the norm is non-negative. From the estimate we get it follows immediately, that (6.2) implies (6.3).

For the first term in (6.6) we have an estimate

$$
\int_{\mathbb{E}} P_{n}^{2}\left|\frac{t z^{\prime}}{\phi}\right|^{2} d m=2 \int P_{n}^{2} d \sigma_{a . c .} \leq 2 \int P_{n}^{2} d \sigma=2 .
$$

Due to Lemma 5.3,

$$
\left\langle b^{n+1} K^{\alpha \mu^{-(n+1)}}, \overline{s\left(t b^{n+1} K^{\alpha \mu^{-(n+1)}}\right)}\right\rangle \rightarrow 0, n \rightarrow \infty,
$$

so,

$$
\begin{aligned}
\left\|b^{n+1} K^{\alpha \mu^{-(n+1)}}-s \overline{\left(t b^{n+1} K^{\alpha \mu^{-(n+1)}}\right)}\right\|^{2} & =1+1-2 \operatorname{Re}\left\langle b^{n+1} K^{\alpha \mu^{-(n+1)}}, \overline{s\left(t b^{n+1} K^{\left.\alpha \mu^{-(n+1)}\right)}\right\rangle}\right. \\
& \rightarrow 2, \quad n \rightarrow \infty .
\end{aligned}
$$

Note, also, that $\overline{\left(\frac{-t z^{\prime}}{\phi}\right)} P_{n}$ is orthogonal to any function of the form $g(t)+s(t) \bar{t} g(\bar{t})$. Therefore,

$$
\left\langle b^{n+1} K^{\alpha \mu^{-(n+1)}}-\overline{s\left(b^{n+1} K^{\alpha \mu^{-(n+1)}}\right)}, \overline{\left(\frac{-t z^{\prime}}{\phi}\right)} P_{n}\right\rangle=2\left\langle b^{n+1} K^{\alpha \mu^{-(n+1)}}, \overline{\left(\frac{-t z^{\prime}}{\phi}\right)} P_{n}\right\rangle .
$$

To evaluate this scalar product we apply the (DCT),

$$
\begin{aligned}
\left\langle b^{n+1} K^{\alpha \mu^{-(n+1)}}, \overline{\left(\frac{-t z^{\prime}}{\phi}\right)} P_{n}\right\rangle & =\int_{\mathbb{E}} b^{n+1} K^{\alpha \mu^{-(n+1)}}\left(\frac{-t z^{\prime}}{\phi}\right) P_{n} \frac{d t}{2 \pi i t} \\
& =\int_{\mathbb{E}} K^{\alpha \mu^{-(n+1)}}\left(\frac{-b^{2} z^{\prime}}{\phi}\right)\left(b^{n} P_{n}\right) \frac{d t}{2 \pi i b} \\
& =\frac{1}{b^{\prime}(0)}\left\{K^{\alpha \mu^{-(n+1)}}\left(\frac{-b^{2} z^{\prime}}{\phi}\right)\left(b^{n} P_{n}\right)\right\}(0) \\
& +\sum_{\zeta_{l} \in Z}\left\{b^{n+1} K^{\alpha \mu^{-(n+1)}}\left(\frac{-z^{\prime}}{\phi^{\prime}}\right) P_{n}\right\}\left(\zeta_{l}\right) .
\end{aligned}
$$

Since $(b z)(0)=1$, we have

$$
\frac{1}{b^{\prime}(0)}\left\{K^{\alpha \mu^{-(n+1)}}\left(\frac{-b^{2} z^{\prime}}{\phi}\right)\left(b^{n} P_{n}\right)\right\}(0)=\frac{K^{\alpha \mu^{-(n+1)}}(0)}{p_{0} \ldots p_{n} \phi(0)}
$$

Let us show that the last term in (6.9) tends to 0. Indeed,

$$
\begin{aligned}
& \sum_{\zeta_{l} \in Z}\left|b^{n+1} K^{\alpha \mu^{-(n+1)}} P_{n} \frac{\left(-z^{\prime}\right)}{\phi^{\prime}}\right|=\sum_{\zeta_{l} \in Z}\left|P_{n}\right|\left|\frac{b^{n+1} K^{\alpha \mu^{-(n+1)}}}{\psi}\right| \frac{\left(-z^{\prime}\right) \psi}{\phi^{\prime}} \\
\leq & \sqrt{\sum_{\zeta_{l} \in Z}\left|P_{n}\right|^{2} \sigma_{l}} \sqrt{\sum_{\zeta_{l} \in Z}\left|\frac{b^{n+1} K^{\alpha \mu^{-(n+1)}}}{\psi}\right|^{2} \sigma_{l} .}
\end{aligned}
$$


Since

$$
\sum_{x_{l} \in X}\left|P_{n}\right|^{2} \sigma_{l} \leq \int\left|P_{n}\right|^{2} d \sigma=1,
$$

using Lemma 4.3 and the definition of the measure $\nu$, we get

$$
\sum_{\zeta_{l} \in Z}\left\{b^{n+1} K^{\alpha \mu^{-(n+1)}}\left(\frac{-z^{\prime}}{\phi^{\prime}}\right) P_{n}\right\}\left(\zeta_{l}\right) \rightarrow 0 .
$$

Substituting $(6.7) \ldots(6.10)$ in (6.6) we obtain

$$
\begin{aligned}
& 2-4 \frac{K^{\alpha \mu^{-(n+1)}}(0)}{p_{0} \ldots p_{n} \phi(0)}+2+o(1) \\
\geq & \left\|\overline{\left(\frac{-t z^{\prime}}{\phi}\right)} P_{n}-\left\{b^{n+1} K^{\alpha \mu^{-(n+1)}}-s \overline{\left(t b^{n+1} K^{\alpha \mu^{-(n+1)}}\right)}\right\}\right\|^{2} \geq 0,
\end{aligned}
$$

and thus the lemma is proved.

Lemma 6.2. Assume that

$$
\|h(\zeta, P ; \sigma)\| \leq C\|P\|_{L_{d \sigma}^{2}},
$$

and that the assumptions of the previous Theorem are satisfied. Then

$$
\lim _{n \rightarrow \infty} \frac{K^{\alpha \mu^{-(n+1)}}(0)}{p_{0} \ldots p_{n} \phi(0)}=1
$$

Proof. Let $h_{n}(\zeta)=h_{n}\left(\zeta, P_{n} ; \sigma\right)$. Then

$$
\overline{\left(\frac{-t z^{\prime}}{\phi}\right)} P_{n}(z(t))=h_{n}-s \bar{t} h_{n}(\bar{t})
$$

where $s=\phi \overline{(t \phi)}$. Multiplying this identity by $b^{n+1} K^{\alpha \mu^{-(n+1)}}$, we get (see $\left.(6.9),(6.10)\right)$

$$
\frac{K^{\alpha \mu^{-(n+1)}}(0)}{p_{0} \ldots p_{n} \phi(0)}+o(1)=\left\langle h_{n}, b^{n+1} K^{\alpha \mu^{-(n+1)}}\right\rangle-\left\langle s \bar{t} h_{n}(\bar{t}), b^{n+1} K^{\alpha \mu^{-(n+1)}}\right\rangle
$$

Using the reproducing property of $K^{\alpha \mu^{-(n+1)}}$ and (3.14) we get

$$
\left\langle h_{n}, b^{n+1} K^{\alpha \mu^{-(n+1)}}\right\rangle=\frac{p_{0} \cdots p_{n} \phi(0)}{K^{\alpha \mu^{-(n+1)}}(0)}
$$

and due to Lemma 5.3 we have

$$
\begin{aligned}
\left|\left\langle s \bar{t} h_{n}(\bar{t}), b^{n+1} K^{\alpha \mu^{-(n+1)}}\right\rangle\right| & =\left|\left\langle\bar{t} h_{n}(\bar{t}), P_{-}\left(\alpha^{-1}\right)\left\{\bar{s} b^{n+1} K^{\alpha \mu^{-(n+1)}}\right\}\right\rangle\right| \\
& \leq\left\|h_{n}\right\|\left\|P_{-}\left(\alpha^{-1}\right)\left\{\bar{s} b^{n+1} K^{\alpha \mu^{-(n+1)}}\right\}\right\| \rightarrow 0, n \rightarrow \infty
\end{aligned}
$$


Thus (6.11) is of the form,

$$
\frac{K^{\alpha \mu^{-(n+1)}}(0)}{p_{0} \ldots p_{n} \phi(0)}+o(1)=\frac{p_{0} \ldots p_{n} \phi(0)}{K^{\alpha \mu^{-(n+1)}}(0)}+o(1)
$$

and the lemma is proved.

Proof of (6.2) and (6.3). Let $r_{0}(z)=r_{0}\left(z ; X \cup X^{(\tau)}\right)$ be a function of the form (5.1), associated with the given set of zeros and poles. For $0<\eta<1$, put

$$
r_{\eta}(z)=\eta r(z)+(1-\eta) r_{0}(z)
$$

Note that for an arbitrary $\eta$ it is also the Stieltjes function with the same set of zeros and poles as $r$. In what follows all functions and coefficients, related to the function $r_{\eta}(z)$ have the same subscript $\eta$. We need some facts concerning these objects.

If $P_{n, \eta}(z)=\frac{z^{n}}{p_{0, \eta} \ldots p_{n, \eta}}+\ldots$ is the orthonormal polynomial with respect to $\sigma_{\eta}$, then

$$
\begin{aligned}
p_{0}^{2} \ldots p_{n}^{2} & =\inf _{\left\{P(z)=z^{n}+\ldots\right\}} \int P^{2} d \sigma \\
& \leq \int\left|p_{0, \eta} \ldots p_{n, \eta} P_{n, \eta}\right|^{2} d \sigma \\
& \leq \frac{1}{\eta} \int\left|p_{0, \eta} \ldots p_{n, \eta} P_{n, \eta}\right|^{2} d \sigma_{\eta} \\
& =\frac{1}{\eta} p_{0, \eta}^{2} \ldots p_{n, \eta}^{2} .
\end{aligned}
$$

Further, since

$$
\frac{1}{\left|\phi_{\eta}\right|^{2}}=\frac{\eta}{|\phi|^{2}}+\frac{1-\eta}{\left|\phi_{0}\right|^{2}}
$$

the function $\log \left|\phi_{\eta}\right|^{2}$ converges to $\log |\phi|^{2}$ in $L^{1}$, as $\eta \rightarrow 1$. Therefore,

$$
\alpha_{\eta} \rightarrow \alpha \quad \text { and } \quad \phi_{\eta}(0) \rightarrow \phi(0), \quad \eta \rightarrow 1
$$

The measure $d \sigma_{\eta}^{(\tau)}$ is absolutely continuous on $E$. Indeed,

$$
\left|\frac{1}{r_{\eta}}\right| \leq\left|\frac{1}{\operatorname{Im} r_{\eta}}\right| \leq \frac{1}{1-\eta}\left|\frac{1}{\operatorname{Im} r_{0}}\right| \leq \frac{1}{1-\eta}\left|\operatorname{Im} \frac{1}{r_{0}}\right| .
$$

And since $-\operatorname{Im} \frac{1}{r_{0}} d x$ is integrable we can apply Lemma 2.4. Simultaneously we have an estimate

$$
d \sigma_{\eta}^{(\tau)} \leq \frac{1}{1-\eta} d \sigma_{0}^{(\tau)} \text { on } E
$$

Since $\sigma_{l}^{(\tau)}=1 / r^{\prime}\left(x_{l}^{(\tau)}\right)$, and

$$
r_{\eta}^{\prime}\left(x_{l}^{(\tau)}\right)=\eta r^{\prime}\left(x_{l}^{(\tau)}\right)+(1-\eta) r_{0}^{\prime}\left(x_{l}^{(\tau)}\right),
$$


we also have

$$
\sigma_{l, \eta}^{(\tau)} \leq \frac{1}{1-\eta} \sigma_{l, 0}^{(\tau)}
$$

So,

$$
d \sigma_{\eta}^{(\tau)} \leq \frac{1}{1-\eta} d \sigma_{0}^{(\tau)}
$$

Next, let us put

$$
-\frac{1}{r_{\eta, \eta_{1}}}=-\frac{\eta_{1}}{r_{\eta}}-\frac{1-\eta_{1}}{r_{0}}
$$

Then, due to (6.14),

$$
\left(1-\eta_{1}\right) d \sigma_{0}^{(\tau)} \leq d \sigma_{\eta, \eta_{1}}^{(\tau)} \leq\left[\frac{\eta_{1}}{1-\eta}+\left(1-\eta_{1}\right)\right] d \sigma_{0}^{(\tau)} .
$$

Now to this measure we can apply Lemma 5.2 and Lemma 6.2. Therefore,

$$
\lim _{n \rightarrow \infty} \frac{K^{\alpha_{\eta, \eta_{1}} \mu^{-(n+1)}}(0)}{p_{0, \eta, \eta_{1}} \ldots p_{n, \eta, \eta_{1}} \psi_{\eta, \eta_{1}}(0)}=1 \text {. }
$$

We remind that the functions $\psi$ and $\phi$ have the same character and that $\phi(0)=\psi(0)$. As above in (6.13), since $\log \left|\psi_{\eta, \eta_{1}}\right| \rightarrow \log \left|\psi_{\eta}\right|$ in $L^{1}\left(\eta_{1} \rightarrow 1\right)$, we have

$$
\alpha_{\eta, \eta_{1}} \rightarrow \alpha_{\eta} \quad \text { and } \quad \psi_{\eta, \eta_{1}}(0) \rightarrow \psi_{\eta}(0), \quad \eta_{1} \rightarrow 1
$$

At last, as in (6.12) we obtain

$$
p_{0, \eta, \eta_{1}}^{2} \ldots p_{n, \eta, \eta_{1}}^{2} \geq \eta_{1} p_{0, \eta}^{2} \ldots p_{n, \eta}^{2} \geq \eta_{1} \eta p_{0}^{2} \ldots p_{n}^{2} .
$$

Since $K^{\alpha}(0)$ depends continuously on $\alpha$, using (6.13) and (6.16), for arbitrary $\epsilon>0$, we can chose $\eta, \eta_{1}$ so close to 1 , that $\forall n$,

$$
\frac{K^{\alpha \mu^{-(n+1)}}(0)}{\phi(0)} \geq \frac{K^{\alpha_{\eta, \eta_{1}} \mu^{-(n+1)}}(0)}{\psi_{\eta, \eta_{1}}(0)}(1-\epsilon) .
$$

Using (6.17), we get

$$
\frac{K^{\alpha \mu^{-(n+1)}}(0)}{p_{0} \ldots p_{n} \phi(0)} \geq \sqrt{\eta \eta_{1}} \frac{K^{\alpha_{\eta, \eta_{1}} \mu^{-(n+1)}}(0)}{p_{0, \eta, \eta_{1}} \ldots p_{n, \eta, \eta_{1}} \psi_{\eta, \eta_{1}}(0)}(1-\epsilon) .
$$

Then, due to (6.15), we get

$$
\varliminf_{n \rightarrow \infty} \frac{K^{\alpha \mu^{-(n+1)}}(0)}{p_{0} \ldots p_{n} \phi(0)} \geq 1 .
$$

Together with (6.5) this finishes the proof of (6.2), and due to Lemma 6.1, (6.3) is also proved. 
Proof of (6.4). We rewrite (6.3) in terms of analytic functions. Taking into account, that $\overline{\left(t K^{\left.\alpha \mu^{-(n+1)}\right)}\right.}=\frac{K^{\alpha^{-1} \mu^{n+2}}}{b}$, we have

$$
g_{n}:=\frac{\left(-z^{\prime}\right)}{\phi} P_{n}-\left\{b^{-(n+2)} K^{\alpha^{-1} \mu^{n+2}}-\bar{s} b^{(n+1)} K^{\alpha \mu^{-(n+1)}}\right\} \rightarrow 0 \quad \text { in } L_{d m \mid \mathbb{E}}^{2} .
$$

In a standard way, using reproducing kernel, we get

$$
\left|\left\langle g_{n}, \overline{\left(b^{n+2} B_{X}\right)} k^{\alpha_{\text {out }}^{-1} \mu^{n+2}}(t, \zeta)\right\rangle\right| \leq\left\|g_{n}\right\| \sqrt{k_{\text {out }}^{\alpha^{-1} \mu^{n+2}}(\zeta, \zeta)},
$$

moreover,

$$
\begin{aligned}
& \left\langle\frac{\left(-z^{\prime}\right)}{\phi} P_{n}-b^{-(n+2)} K^{\alpha^{-1} \mu^{n+2}}, \overline{\left(b^{n+2} B_{X}\right)} k^{\alpha_{\text {out }}^{-1} \mu^{n+2}}(t, \zeta)\right\rangle \\
= & \left(\frac{\left(-z^{\prime}\right) b^{2}}{\phi^{\text {out }}} b^{n} P_{n}-B_{X} K^{\alpha^{-1} \mu^{n+2}}\right)(\zeta) .
\end{aligned}
$$

Therefore, our goal is to evaluate the scalar product

$$
\left\langle b^{n+1} K^{\alpha \mu^{-(n+1)}}, s \overline{\left(b^{n+2} B_{X}\right)} k^{\alpha_{\text {out }}^{-1} \mu^{n+2}}(t, \zeta)\right\rangle .
$$

We claim that this product tends to 0 uniformly with respect to $\zeta$.

Let $w$ be an inner function from $H^{\infty}(\Gamma)$, for example, $w=b \Delta^{\mu^{-1}}$. Then

$$
\overline{\left(\frac{w-w(\zeta)}{1-w \overline{w(\zeta)}}\right)} k^{\alpha_{\text {out }}^{-1} \mu^{n+2}}(t, \zeta) \in L_{d m \mid \mathbb{E}}^{2} \ominus A_{1}^{2}\left(\Gamma, \alpha_{\text {out }}^{-1} \mu^{n+2}\right),
$$

and

$$
\left\|\overline{B_{X_{-}} b^{n+2}} \overline{\left(\frac{w-w(\zeta)}{1-w \overline{w(\zeta)}}\right)} k^{\alpha_{\text {out }}^{-1} \mu^{n+2}}(t, \zeta)\right\|=\sqrt{k_{\text {out }}^{\alpha^{-1} \mu^{n+2}}(\zeta, \zeta)} .
$$

For a fixed compact $\Xi \subset \overline{\mathbb{C}} \backslash E$, put $f(t, z)=s \frac{w-w(\zeta)}{1-w \overline{w(\zeta)}}, z(\zeta)=z \in \Xi$. This is a $L^{\infty}\left(\alpha^{2}\right)-$ valued continuous function on $\Xi$. Since

$$
\begin{aligned}
& \left\langle b^{n+1} K^{\alpha \mu^{-(n+1)}}, s \overline{\left(b^{n+2} B_{X}\right)} k^{\left.\alpha_{\text {out }}^{-1} \mu^{n+2}(t, \zeta)\right\rangle}\right. \\
= & \left\langle b^{n+1} K^{\alpha \mu^{-(n+1)}}, f(t, z) \overline{B_{X} b^{n+2}} \overline{\left(\frac{w-w(\zeta)}{1-w \overline{w(\zeta)}}\right)} k^{\left.\alpha_{\text {out }}^{-1} \mu^{n+2}(t, \zeta)\right\rangle}\right. \\
= & \left\langle P_{-}\left(\alpha^{-1}\right)\left\{\overline{f(t, z)} b^{n+1} K^{\alpha \mu^{-(n+1)}}\right\}, \overline{B_{X} b^{n+2}} \overline{\left(\frac{w-w(\zeta)}{1-w \overline{w(\zeta)}}\right)} k^{\left.\alpha_{\text {out }}^{-1} \mu^{n+2}(t, \zeta)\right\rangle,}\right.
\end{aligned}
$$

(see (6.20)) we can apply Lemma 5.3 . Together with (6.18), (6.19), (6.21), we obtain the estimate: $\forall \epsilon \exists n_{0}$, such that

$$
\left|\left(\frac{\left(-z^{\prime}\right) b^{2}}{\phi^{\text {out }}} b^{n} P_{n}\right)(\zeta)-\left(B_{X} K^{\alpha^{-1} \mu^{n+2}}\right)(\zeta)\right| \leq \epsilon \sup _{\alpha \in \Gamma^{*}} \sqrt{k^{\alpha}(\zeta, \zeta)}, \quad n \geq n_{0}, z(\zeta) \in \Xi .
$$


Note, that

$$
\sup _{\alpha \in \Gamma^{*}} \frac{\sqrt{k^{\alpha}(\zeta, \zeta)}\left|\phi^{\text {out }}(\zeta)\right|}{\left|\left(b^{2} z^{\prime}\right)(\zeta)\right|}
$$

defines a function in $\overline{\mathbb{C}} \backslash E \equiv \mathbb{D} / \Gamma$, which is uniformly bounded on $\Xi$. Therefore,

$$
\left|b^{n} P_{n}-\frac{B_{X} \phi^{\text {out }}}{\left(-z^{\prime}\right) b^{2}} K^{\alpha^{-1} \mu^{n+2}}\right| \leq C(\Xi) \epsilon
$$

and the theorem is proved.

Proof of Corollary 0.1. In this case $X=\emptyset$, and $X^{(\tau)}$ contains at most one point in each gap. Therefore, due to the lemma of Jones and Marshall (see Sect. 1) condition (6.1) holds automatically. Moreover $\phi$ is an outer function. So, substituting (2.9) in (6.2), (6.3) and (6.4) we get (0.18), (0.20) and (0.19) respectively.

Corollary 6.1. Under the assumptions of the previous Theorem, the recurrence coefficients $\left\{p_{n}\right\},\left\{q_{n}\right\}$ have the following asymptotic behavior

$$
p_{n}-\mathcal{P}\left(\alpha^{-1} \mu^{n+1}\right) \rightarrow 0, \quad n \rightarrow \infty,
$$

and

$$
q_{n-1}-\mathcal{Q}\left(\alpha^{-1} \mu^{n+1}\right) \rightarrow 0, \quad n \rightarrow \infty .
$$

Moreover, $\left\{p_{n}\right\}$ and $\left\{q_{n}\right\}$ are limit (uniformly) almost periodic.

Proof. As it was already shown, (we still assume, that $(b z)(0)=1$ )

$$
\frac{p_{1} \ldots p_{n} \phi(0)}{K^{\alpha \mu^{-(n+1)}}(0)} \rightarrow 1, \quad n \rightarrow \infty
$$

Therefore,

$$
\lim _{n \rightarrow \infty} \frac{p_{1} \ldots p_{n} \phi(0)}{K^{\alpha \mu^{-(n+1)}}(0)} \frac{K^{\alpha \mu^{-n}}(0)}{p_{1} \ldots p_{n-1} \phi(0)}=\lim _{n \rightarrow \infty} \frac{p_{n}}{\mathcal{P}\left(\alpha \mu^{-(n+1)}\right)}=1
$$

It is a characteristic property of domains of Widom type, that

$$
0<\inf _{\alpha \in \Gamma^{*}} K^{\alpha}(0) \leq \sup _{\alpha \in \Gamma^{*}} K^{\alpha}(0)<\infty .
$$

Therefore, (6.25) implies

$$
p_{n}-\mathcal{P}\left(\alpha \mu^{-(n+1)}\right) \rightarrow 0, \quad n \rightarrow \infty
$$

Recall, that $K^{\alpha}(0) K^{\alpha^{-1} \mu}(0)=b^{\prime}(0)$, hence

$$
\mathcal{P}(\alpha)=\frac{K^{\alpha}(0)}{K^{\alpha \mu}(0)}=\frac{K^{\alpha^{-1}}(0)}{K^{\alpha^{-1} \mu}(0)}=\mathcal{P}\left(\alpha^{-1}\right) .
$$


So we can rewrite $(6.26)$ in the form $(6.22)$.

To prove (6.23) we use (6.4). Since,

$$
\begin{aligned}
& P_{n} b^{n}-\frac{\phi}{\left(-z^{\prime}\right) b^{2}} K^{\alpha^{-1} \mu^{n+2}} \rightarrow 0, \\
& (z b) P_{n-1} b^{n-1}-\frac{\phi}{\left(-z^{\prime}\right) b^{2}}(z b) K^{\alpha^{-1} \mu^{n+1}} \rightarrow 0, \\
& \left(p_{n}-\mathcal{P}\left(\alpha^{-1} \mu^{n+1}\right)\right) \frac{\phi}{\left(-z^{\prime}\right) b^{2}} K^{\alpha^{-1} \mu^{n+2}} \rightarrow 0, \quad n \rightarrow \infty,
\end{aligned}
$$

we have

$$
\left(z P_{n-1}-p_{n} P_{n}\right) b^{n}-\frac{\phi}{\left(-z^{\prime}\right) b^{2}}\left(z b K^{\alpha^{-1} \mu^{n+1}}-\mathcal{P}\left(\alpha^{-1} \mu^{n+1}\right) K^{\alpha^{-1} \mu^{n+2}}\right) \rightarrow 0, \quad n \rightarrow \infty
$$

Then, due to the maximum principle for analytic functions,

$$
\left(z P_{n-1}-p_{n} P_{n}\right) b^{n-1}-\frac{\phi}{\left(-z^{\prime}\right) b^{3}}\left(z b K^{\alpha^{-1} \mu^{n+1}}-\mathcal{P}\left(\alpha^{-1} \mu^{n+1}\right) K^{\alpha^{-1} \mu^{n+2}}\right) \rightarrow 0, \quad n \rightarrow \infty
$$

(note, that this function has no pole at the origin). Using the recurrence relations and putting $\zeta=0$, we get

$$
\left\{\frac{q_{n-1}}{p_{1} \ldots p_{n-1}}-\frac{\phi(0)}{b^{\prime}(0)} \mathcal{Q}\left(\alpha^{-1} \mu^{n+1}\right) K^{\alpha^{-1} \mu^{n+1}}(0)\right\} \rightarrow 0, \quad n \rightarrow \infty .
$$

Using again the identity $K^{\alpha}(0) K^{\alpha^{-1} \mu}(0)=b^{\prime}(0)$ and (6.24), we get (6.23).

\section{REFERENCES}

1. N.I. Akhiezer, Orthogonal polynomials on several intervals., Soviet Math. Dokl. 1 (1960), 989-992.

2. N.I. Akhiezer, Elements of the theory of elliptic functions, American Mathematical Society, Providence, RI, 1990.

3. N.I. Akhiezer and Yu.Ya. Tomchuk, On the theory of orthogonal polynomials over several intervals, Dokl. Akad. Nauk SSSR 138 (1961), 743-746. (Russian)

4. A.I. Aptekarev, Asymptotic properties of polynomials orthogonal on a system of contours, and periodic motions of Toda chains, Mat. Sb. (N.S.) 125(167) (1984), 231-258; English transl. in Math. USSR Sb., 53 (1986), 233-260.

5. S.N. Bernstein, Sur les polynomes orthogonaux relatifs á un segment fini, I, Journ. de math. pures et appl. 9 (1930), 127-177.

6. L. Carleson, On $H^{\infty}$ in multiply connected domains, Conference on harmonic analysis in honor Antoni Zygmund. (eds. W Beckner, et al.). vol. II., Wadsworth, 1983, pp. 349-372.

7. R. Carmona and J. Lacroix, Spectral Theory of Random Schrödinger Operators, Birkhäuser, 1990.

8. W. Craig, The trace formula for Schrödinger operators on the line, Commun. Math. Phys. 126 (1989), 379-408.

9. H.L. Cycon, R.G. Froese, W. Kirsch, B. Simon, Schrödinger Operators with Application to Quantum Mechanics and Global Geometry, Springer-Verlag, Berlin, 1987.

10. P. Deift, Orthogonal polynomials and random matrices: a Riemann-Hilbert approach, Courant Lecture Notes in Math., Vol. 3, Courant Institute of Math. Sciences, 1999. 
11. B.A. Dubrovin, I.M. Krichever and S.P. Novikov, Dynamical systems. IV, Springer-Verlag, Berlin, 1990, pp. 173-280.

12. J. Garnett, Bounded analytic functions, Academic press, 1981.

13. J.S. Geronimo and W. Van Assche, Approximating the weight function for orthogonal polynomials on several intervals, J. Approx. Theory 65 (1991), 341-371.

14. M. Hasumi, Hardy Classes on Infinitely Connected Riemann Surfaces, Lecture Notes in Math. 1027, Spinger Verlag, Berlin and New York, 1983.

15. P. Jones, Some problems in complex analysis, The Bieberbach Conjecture. Proc. of the Symp. on the Occasion of the Proof (eds., A. Baernstein and D. Drasin), Amer. Math. Soc., Providence, RI, 1986, pp. 105-108.

16. P. Jones and D. Marshall, Critical points of Green's function, harmonic measure, and the Corona problem, Arkiv för Matematik 23 (1985), 281-314.

17. D.S. Lubinsky, Asymptotics of Orthogonal Polynomials: some old, some new, some identities, to appear in Proc. of the Conf. on "Rational Approximation", (eds., A. Cuyt, B. Verdonk), Antwerp June 6-12, 1999, Kluwer Academic press.

18. A. Magnus, Recurrence coefficients for orthogonal polynomials on connected and nonconnected sets, Padé approximation and its applications (Proc. Conf., Univ. Antwerp, Antwerp, 1979), Springer, Berlin, 1979, pp. 150-171.

19. V.A. Marchenko, Sturm-Liouville Operators and Applications, Birkhäuser Verlag, 1986.

20. R. Nevanlinna, Analytic Functions, Springer Verlag, Berlin, 1970.

21. E.M. Nikishin, The discrete Sturm-Liouville operator and some problems of function theory, Trudy Sem. Petrovsk. 10 (1984), 3-77 (Russian); English transl. in Soviet Math., 35 (1987), 2679-2744.

22. E.M. Nikishin and V.N. Sorokin, Rational approximations and orthogonality, American Mathematical Society, Providence, RI, 1991.

23. L. Pastur and A. Figotin, Spectra of Random and Almost-Periodic Operators, Springer Verlag, Berlin, 1986.

24. F. Peherstorfer, Elliptic orthogonal and extremal polynomials, Proc. London Math. Soc. (3) 70 (1995), 605-624.

25. F. Peherstorfer, On Bernstein-Szegö orthogonal polynomials on several intervals, SIAM J. Math. Anal. 21 (1990), 461-482.

26. F. Peherstorfer, On Bernstein-Szegö orthogonal polynomials on several intervals II: orthogonal polynomials with periodic reccurence coefficients, J. Approx. Theory 64 (1991), 123-161.

27. F. Peherstorfer and R. Steinbauer, On polynomials orthogonal on several intervals, Ann. Numer. Math. 2 (1995), 353-370.

28. Ch. Pommerenke, On the Green's function of Fuchsian groups, Ann. Acad. Sci. Fenn. 2 (1976), 409427.

29. M. Sodin and P. Yuditskii, Almost periodic Jacobi matrices with homogeneous spectrum, infinite dimensional Jacobi inversion, and Hardy spaces of character-automorphic functions, Journ. of Geom. Analysis 7 (1997), 387-435.

30. G. Szegö, Orthogonal polynomials, 4th ed., Amer. Math. Soc. Colloq. Publ., Vol. 23, Amer. Math. Soc., Providence, R.I., 1975.

31. M. Toda, Theory of nonlinear lattices, Springer-Verlag, Berlin, 1989.

32. Yu. Tomchuk, Orthogonal polynomials over a system of intervals on the real line, Zap. Fiz.-Mat. Fak. i Khar'kov. Mat. Obšč. (4) 29 (1963), 93-128. (Russian)

33. W. Van Assche, Asymptotics for Orthogonal Polynomials, Lecture Notes in Math. 1265, Springer, Berlin, 1987.

34. H. Widom, Extremal polynomials associated with a system of curves in the complex plane, Adv. Math. 3 (1969), 127-232.

35. H. Widom, The maximum principle for multiple valued analytic functions, Acta Math. 126 (1971), 63-81.

36. P. Yuditskii, Two remarks on Fuchsian groups of Widom type, Oper. Theory Adv. Appl. (to appear).

Institute for Analysis and Computational Mathematics, Johannes Kepler University of Linz, A-4040 Linz, Austria 
E-mail address: Franz.Peherstorfer@jk.uni-linz.ac.at

Dept. of Mathematics, Michigan State University, East Lansing, Mi 48824, USA

E-mail address: yuditski@math.msu.edu 